\title{
Reduced-Order Modeling of Unsteady Aerodynamics Across Multiple Mach Regimes
}

\author{
Torstens Skujins* and Carlos E. S. Cesnik \\ University of Michigan, Ann Arbor, Michigan 48109-2140
}

DOI: $10.2514 / 1 . C 032222$

\begin{abstract}
A reduced-order model for unsteady aerodynamic calculations across a range of Mach regimes based on linear convolution and a nonlinear correction factor is developed. Separate investigations are conducted for the sub-, trans-, and supersonic Mach regimes, and overall good results are seen when reduced-order model results are compared with full-order computational-fluid-dynamics solutions, though the reduced-order model errors tend to decrease as the Mach number increases. To assist reduced-order model construction, the method-of-segments simplified model has been created and tested throughout these same Mach regimes. Finally, a practical example of the reduced-order model's applicability is presented by following a single test case from subsonic up through supersonic flight.
\end{abstract}

\section{Nomenclature}

$C_{d} \quad=\quad$ drag coefficient

$C_{l} \quad=$ lift coefficient

$C_{m}=$ moment coefficient

$d_{i}=$ modal amplitude of $i$ th mode

$f(t) \quad=$ arbitrary input

$f_{c}=$ correction factor

$H(t) \quad=\quad$ unit step response

$k=$ reduced frequency

$M_{\text {sim }}=$ freestream Mach number of test case simulation

$M_{\text {step }}=$ freestream Mach number used for step response computation

$N_{\text {samp }}=$ number of kriging surface sampling points

$y_{\text {conv }}=$ uncorrected linear reduced-order model found via convolution

$y_{\text {corr }}=$ corrected reduced-order model response

$y_{\text {lin }}=$ steady linear response of a certain modal deformation

$y_{\text {nonlin }}=$ steady nonlinear response of a certain modal deformation

$\alpha \quad=$ angle of attack

$\delta=$ correction factor numerical offset value

$\dot{\eta} \quad=$ modal velocity

$\omega=$ natural frequency, $\mathrm{rad} / \mathrm{s}$

\section{Introduction}

$\mathbf{T}$ HE flight envelope for aerospace vehicles will often encompass a range of Mach regimes, thus adding distinct challenges to the development of these vehicles versus those that will fly in much smaller envelopes. For example, for hypersonic vehicles, many of these challenges stem from the tight coupling of vehicle components, including the aerodynamic, structural, and heating loads, engine, and other factors at hypersonic speeds. Designing a controllable vehicle at hypersonic conditions is a hard problem, but neglecting to consider the performance of the vehicle at other, lower flight speeds may lead to controllability issues before attaining hypersonic flight. Because of

Presented as Paper 2012-1708 at the 53rd AIAA/ASME/ASCE/AHS/ASC Structures, Structural Dynamics, and Materials Conference, Honolulu, HI, 23-26 April 2012; received 29 November 2012; revision received 1 April 2014; accepted for publication 14 April 2014; published online 10 September 2014. Copyright (C) 2014 by Torstens Skujins and Carlos E.S. Cesnik. Published by the American Institute of Aeronautics and Astronautics, Inc., with permission. Copies of this paper may be made for personal or internal use, on condition that the copier pay the $\$ 10.00$ per-copy fee to the Copyright Clearance Center, Inc., 222 Rosewood Drive, Danvers, MA 01923; include the code 1542-3868/14 and \$10.00 in correspondence with the CCC.

*Graduate Research Assistant, Department of Aerospace Engineering; tskujins@umich.edu. Member AIAA.

${ }^{\dagger}$ Professor, Department of Aerospace Engineering, 1320 Beal Avenue, 3024 FXB; cesnik@umich.edu. Fellow AIAA. this, it is vital to consider early on in the design process the controllability of the vehicle, when modifications can still be made with relative ease. In [1], a reduced-order model (ROM) of super/hypersonic unsteady aerodynamics has been created to integrate within a full six-degree-of-freedom hypersonic-vehicle controls-simulation framework. Results obtained from this model have compared favorably with full-order solutions at hypersonic speeds. The reducedorder modeling methodology has been chosen due to its combination of accuracy and computational efficiency. As in other ROMs, the model extracts data from a limited number of upfront full-order computational fluid dynamics (CFD) simulations and uses those data to construct models that run in a computationally efficient manner, up to several orders of magnitude faster than the full-order solutions.

However, the controllability of an aerospace vehicle cannot be assessed solely by considering its response at its highest-speed Mach regime. During the course of a single flight, the vehicle will take off in the subsonic Mach regime and accelerate through the transonic and supersonic regimes on the way up to its maximum velocity. Each of these regimes presents modeling challenges that must be accounted for in the aerodynamic model, a sampling of which are shown in Fig. 1. When conducting a controls simulation, it is desirable to use only one single aerodynamic model throughout the course of the entire simulation. This avoids the inevitable computational expense of switching one model out for another at various times during the simulation as well as the potential for increased model errors and mismatches at the applicability boundaries of the individual models when one model needs to be swapped out for another one. Thus, a single aerodynamic model allows for a single representative form of the aerodynamic loads to be used, thereby increasing the efficiency of the entire simulation.

In this research, a convolution type of ROM [2,3] is selected due to the ease of implementation within the CFD code framework as well as the fact that it has previously been shown to perform well in aerodynamic modeling applications. Convolution methods take advantage of Duhamel's integral to combine a system's unit step or impulse response with some arbitrary motion to calculate a quantity of interest for a linear system. Along with Volterra series [4,5], which is the nonlinear analog of convolution with the addition of higherorder nonlinear terms, convolution-based methods have a rich literature in terms of development and application to unsteady aerodynamics. Early efforts to apply the Volterra series to aerodynamic problems include Baumann et al. [6], who used Volterra series to compute aircraft flying quality parameters. In his Ph.D. thesis, Silva [2] developed a method to calculate the unsteady aerodynamic loads on a transonic airfoil using first- and second-order Volterra series kernels obtained from CFD simulations. Then, a framework was developed to transform the step/impulse responses into a state-space reduced-order model of the system, in which a certain output is a function of current and past system state variables and system inputs; from this state-space representation, flutter results 
Hypersonic vehicle flight trajectory

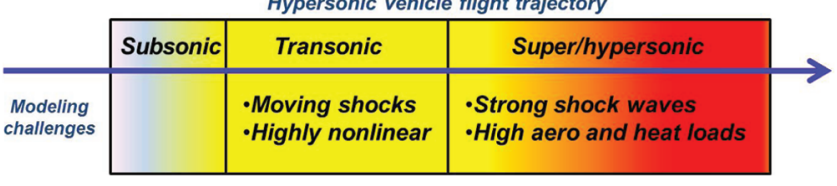

Fig. 1 Sample hypersonic vehicle flight regimes.

could be obtained [7]. In this framework, each elastic mode shape was considered separately, and so separate simulations needed to be conducted for each individual mode shape. This issue can become problematic when the number of mode shapes increases. To remedy this problem, Silva [8] developed a method for the construction of the state-space system that permits the excitation of multiple mode shapes in the course of a single CFD simulation, thus increasing the computational efficiency by decoupling the number of CFD simulations from the number of mode shapes under consideration. Kim [9] and Kim et al. [10] have also researched methods to reduce the number of CFD simulations through the use of a single composite input composed of multiple system inputs for state-space system construction. Gaitonde and Jones [11] developed a method for computing a continuous ROM based on CFD impulse responses, and Allen et al. [12] validated the model against full-order solutions for flutter boundary predictions for a two-dimensional airfoil/controlsurface combination. Singh and Baeder [13] computed indicial responses to changes in angle of attack for a three-dimensional wing and then used the solutions to build numerical databases.

As discussed previously, it is desirable for a ROM to be valid across a range of flight conditions and not only in some narrow range of Mach numbers or other parameters. Thus, research has been conducted to investigate this issue for convolution/Volterra type of ROMs. Lind et al. [14] used Volterra kernels from wind-tunnel test data to compute state-space systems at several different flight conditions, to which a model was then fitted to permit the calculation of quantities of interest away from the specific flight conditions of the test data. Prazenica et al. [15] extrapolated Volterra kernels calculated using flight-test data at certain flight conditions to other, different flight conditions, resulting in a ROM valid for varying parameters. Omran and Newman [16] used interpolation of Volterra series submodels constructed at specific flight conditions to build global piecewise Volterra kernels valid over a much larger range of flight conditions. In another work, the same authors [17] used a nonlinear parameter-varying approach consisting of local Volterra models to be able to account for strong nonlinearities over multiple aircraft flight regions. Other efforts have investigated the numerical integration of the state-space system itself. Silva [18] created a ROM valid over a range of velocities by changing the numerical integration time step.

Skujins and Cesnik [1] focus on the development of the model and how it overcomes the limitations inherent to convolution-type ROMs presented in the literature as well as its specific application to the hypersonic regime. This paper focuses on the application of the model to the sub-, trans-, and supersonic Mach regimes. To start with, the narrow Mach-number region around Mach 1, encompassing the transonic regime, is considered. This regime is characterized by the appearance and motion of shock waves as portions of the flow reach Mach 1, thereby creating nonlinear flowfields and hence further challenges when modeling the aerodynamic loads. Accurate modeling here is important due to the various aeroelastic and aerodynamic phenomena encountered, including the flutter transonic dip as well as a significant increase in the drag as flight speed nears Mach 1. Next, the Mach range of interest is zoomed out to encompass portions of the sub- and supersonic regimes. Finally, a more practical example of ROM usage is given by considering one example test case from subsonic up through supersonic flight.

\section{Reduced-Order Model Formulation}

The reduced-order modeling methodology used in this research is described in detail in [1], and the ROM framework is displayed in Fig. 2. The ROM itself combines linear convolution with a nonlinear

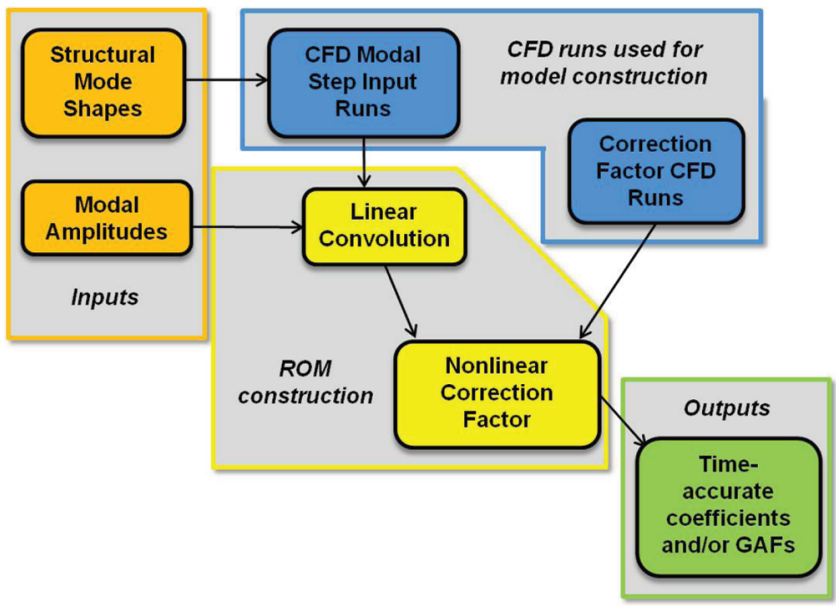

Fig. 2 Overall ROM framework.

correction factor, which accounts for large geometric deformations as well as flight conditions other than the specific set where the ROM is constructed. A summary of the ROM highlights is presented here, and the reader is referred to [1] for a more-detailed description.

\section{A. Linear Convolution}

The linear response of a system to an arbitrary input can be found by combining the system's unit step response with the derivative of the arbitrary input $f(t)$ through the use of Duhamel's integral, displayed as follows in a continuous-time sense $[2,19]$ :

$$
y(t)=f(0) H(t)+\int_{0}^{t} \frac{\mathrm{d} f}{\mathrm{~d} t}(\tau) H(t-\tau) \mathrm{d} \tau
$$

where $H(t)$ is the response of the system to a step input. The step input is zero up through some initial time $t_{0}$ and then 1 for every time step thereafter. In a discrete-time sense, which is the form used in a CFD framework, the integral and step input are as follows [2]:

$$
y[n]=f[0] H[n]+\sum_{k=0}^{n}(u[n]-u[n-1]) H[n-k]
$$

Discrete Unit Step:

$$
u_{\text {step }}[n]=0, \quad n<0 \quad u_{\text {step }}[n]=1, \quad n \geq 1
$$

Duhamel's integral can also be written in terms of the system's unit impulse response, but the improvement of results with the step input is observed and had already been noted by Raveh [20]. In general, convolution can be thought of as a summation of scaled and shifted step/impulse responses. It is this superposition of responses to find the final response that makes convolution a linear concept. The final result after the implementation of the convolution process is the linear ROM response denoted as $y_{\text {conv }}$. In this work, these step responses are computed by conducting CFD runs in which a step input is given to a specific mode shape of the geometry under consideration.

\section{B. Nonlinear Correction Factor}

Initial tests showed that the linear ROM will generally decrease in accuracy as the flight conditions and input amplitudes move away from those used for the step responses, demonstrating the need for some sort of correction term. To obtain the nonlinear corrected ROM response $y_{\text {corr }}$, a correction factor $f_{c}$ is introduced. This quantity is defined as the ratio between the steady linear $\left(y_{\text {lin }}\right)$ and nonlinear $\left(y_{\text {nonlin }}\right)$ responses of a certain configuration due to the modal deformations and flight conditions at a particular instant in time, that is,

$$
f_{c} \equiv \frac{y_{\text {nonlin }}}{y_{\text {lin }}}
$$


To calculate $y_{\text {nonlin }}$, all modal input amplitudes are given to the system simultaneously. The solution is allowed to run until a final steady value is reached; this solution is taken to be $y_{\text {nonlin }}$. To find $y_{\text {lin }}$, each modal amplitude is input to the system separately and allowed to reach a steady value. $y_{\text {lin }}$ is then computed by summing the individual solution values. Skujins and Cesnik [1] provide further details on the procedures for calculating both $y_{\text {lin }}$ and $y_{\text {nonlin }}$.

For a purely linear system, the correction factor value will be 1 . In certain situations, the individual responses used for $y_{\text {lin }}$ calculations will sum to be equal to or very close to zero, resulting in a $y_{\text {lin }}$ value approaching zero and hence an $f_{c}$ value approaching infinity. For these situations, the definition is modified by the addition of an offset term $\delta$ :

$$
f_{c}=\frac{y_{\text {nonlin }}+\delta}{y_{\operatorname{lin}}+\delta}
$$

Note that $\delta$ is placed in the numerator as well as the denominator such that a linear system will still have a correction factor value of 1 .

With this correction factor definition in place, the corrected ROM value $y_{\text {corr }}$ is calculated by

$$
y_{\text {corr }}=\left(f_{c}\right) y_{\text {conv }}=\left(\frac{y_{\text {nonlin }}}{y_{\text {lin }}}\right) y_{\text {conv }}
$$

If an offset term is used, the definition becomes

$$
y_{\text {corr }}=f_{c}\left(y_{\text {conv }}+\delta\right)-\delta
$$

This leads to the basic correction factor assumption that the ratio of the steady response values at a particular time step $t$ will be equal to the ratio of unsteady response values at that particular time step, namely

$$
\left.\frac{y_{\text {nonlin }}}{y_{\text {lin }}}\right|_{t}=\left.\frac{y_{\text {corr }}}{y_{\text {conv }}}\right|_{t}
$$

The errors between the ROM and full-order CFD simulation results will characterize the validity of this assumption, as conditions resulting in larger ROM errors will show likely areas where this assumption breaks down.

Now that the correction factor has been defined, the challenge is to find its value over the entire parameter space, which in this work consists of modal amplitudes and Mach number. To do so, individual correction factor CFD runs are conducted to find the value $y_{\text {nonlin }}$ at those particular points. Then, with these $y_{\text {nonlin }}$ values in hand, the correction factor at each of those points is calculated. Finally, a kriging $[21,22]$ surface is constructed with these sampling point values, which permits the correction factor value at any point in the parameter space to be obtained.

\section{Sampling Point Determination}

To determine the best number and location of the correction factor kriging surface sampling points, simplified models are used for this process, which is shown in Fig. 3. First, an initial number of sampling points is selected using Latin hypercube sampling [23] . Then, rather than using CFD, the simplified model is used to calculate $y_{\text {nonlin }}$ at each of these points. Next, a kriging surface is constructed, and an additional point is placed at the location of the surface's maximum error location. The surface is reconstructed, the maximum error is recomputed, and the process continues until some user-defined stopping criterion has been met. Note that the simplified models are used here due to the fact that the computational time to complete this process using CFD would be large because the sampling points after the initial Latin hypercube values are determined in a sequential nature and thus cannot be computed in parallel, i.e., the $(i+1)$ th sampling point cannot be determined until the maximum error for the kriging surface with $i$ points has been calculated.

\section{Simplified Model Selection}

The selection of the specific simplified model does tend to be flight-regime specific. In [1], piston theory was chosen for the studies in the super/hypersonic Mach regimes. However, the lower-speed flight regimes in this work are outside of piston theory's applicability boundaries. Thus, some other model needs to be selected. To fulfill this need, the method of segments has been developed and is presented next.

\section{Method of Segments}

The method of segments (MoS) is a simplified model that has been been created to efficiently calculate correction factor values at locations throughout the parameter space without requiring a separate CFD run at each of the locations. The basic idea is that, when the wing is in an elastically deformed position, it can be approximated as a series of chordwise-rigid segments along the span that are at different angles of attack, as shown in Fig. 4 .

Because the correction factor methodology relies on the steadystate coefficients after a certain modal deformation has been input, the lift and drag at each of the chordwise segments along the span are found through the use of steady rigid CFD simulations conducted at varying angles of attack and Mach numbers. Although the individual segments will also undergo a plunge motion in addition to pitching motion during elastic deformations, these plunge motions are neglected here due to the steadiness of the CFD solutions being found. The specific steps to the method, shown graphically in Fig. $\underline{5}$, are as follows:

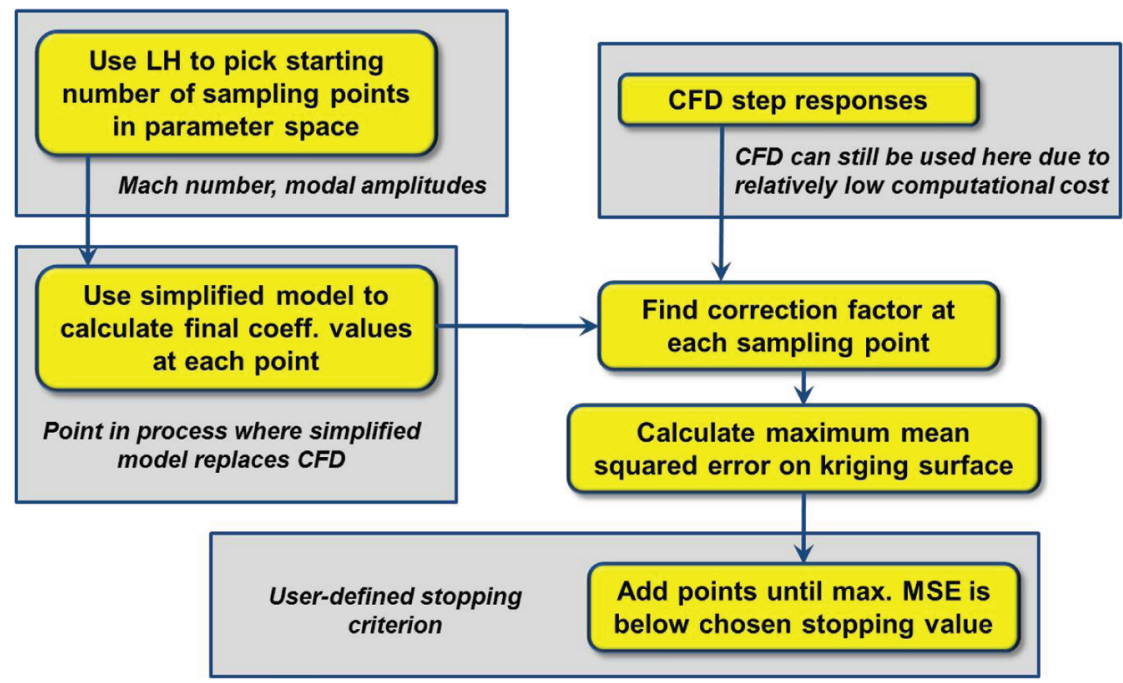

Fig. 3 Sampling point determination methodology. 


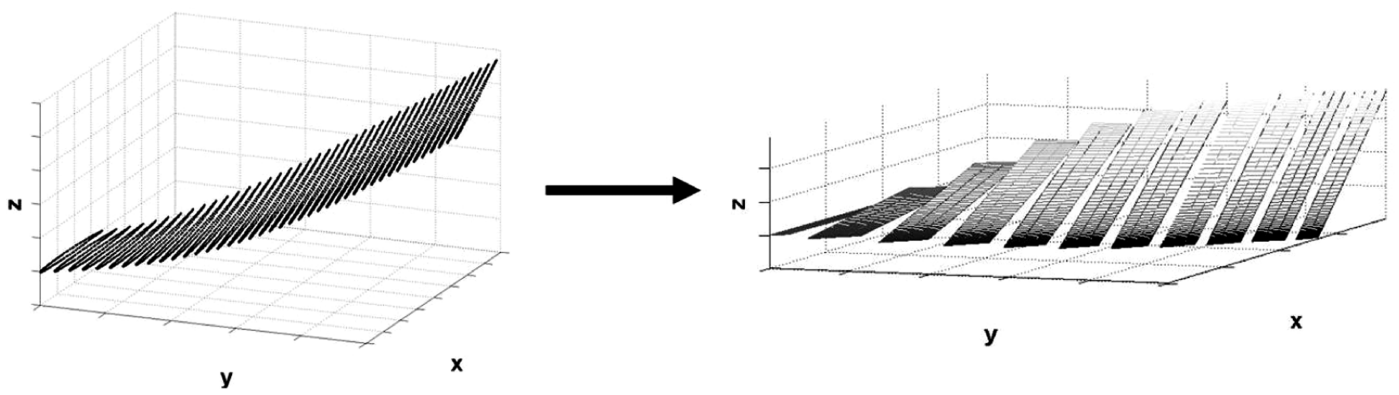

Fig. 4 AGARD wing divided into segments.

1) Divide the wing into chordwise segments along the span, which are assumed to be rigid in the chordwise direction (e.g., the AGARD 445.6 wing has been divided into 11 of these chordwise-rigid segments).

2) Conduct steady, rigid CFD runs throughout the parameter space, which consists of Mach number and angle of attack. Note that one CFD run here will consist of finding the steady solution for one rigidbody angle of attack at a specific Mach number. From this run, the lift and drag for each segment at those conditions is tracked. Thus, one CFD run provides information regarding all of the segments. The parameter space dimensionality will remain at two regardless of how many modes are being considered. Because of this, the total number of runs remains relatively low, and steady runs are computationally cheaper than unsteady ones.

3) Track the lift and drag forces as well as the pitching moment on each of the chordwise segments, taking into consideration the spanwise width of each segment. Construct separate kriging surfaces for the force and moment quantities at each of the segments. Steps 13 are all performed upfront, before the ROM simulations.

4) For a certain wing deformation at a particular time step in a simulation, calculate the local angle of attack at each wing segment.

5) Pick the lift, drag, and moment off the kriging surfaces for each segment corresponding to the specific Mach number and local angle of attack; sum them together to find the lift, drag, and moment for the entire wing.

6) Calculate the coefficients for the wing. These values can then be used to formulate the correction factor for that particular set of parameters.

The accuracy of MoS is the subject of several investigations presented later in this paper.

\section{Reduced-Order Model Process}

The overall ROM process is shown graphically in Fig. 2. To begin with, two separate sets of inputs are given to the ROM. The first are the structural mode shapes of the vehicle or geometry under consideration. These mode shapes are used for both types of CFD runs for model construction. The first type is the modal step input runs, in which the step response for each mode shape is found through an individual CFD simulation. The second type of CFD runs are those used for correction factor computations. Because the mode shapes are known a priori, all CFD runs can be completed upfront; thus, once the model has been constructed, the full-order solutions are removed from the ROM loop, allowing the model to run orders of magnitude faster than if the full-order solutions were still required during the simulation.

The next inputs are the modal amplitudes and flight conditions, given at each time step during the simulation; the $i$ th modal amplitude $d_{i}$ is given in terms of multiples of the unit step input amplitude used for linear convolution. These modal amplitudes are convolved with the modal step input runs to give the linear, uncorrected ROM results. Then, the nonlinear correction factor is added to give the final, corrected ROM results. In this work, the outputs are the time-accurate coefficients; however, other quantities, such as the generalized aerodynamic forces (GAFs), could be used as well with the exact same ROM framework.

\section{E. Reduced-Order Model Variations}

To assess both the ROM methodology itself as well as the full potential of MoS, four different ROM variations have been constructed, as shown in Table 1 . Rather than simply being limited to sampling point determination, two of these ROMs use MoS to directly compute the correction factor values used for the ROM. The first ROM, denoted from here on as ROM A, takes advantage of the relative computational efficiency of $\mathrm{MoS}$ by directly calculating the MoS correction factor value at each time step throughout the simulation without the computation of any correction factor kriging surfaces. Thus, this ROM eliminates any uncertainties based on the kriging surface fit of the data. The second MoS-based ROM, denoted

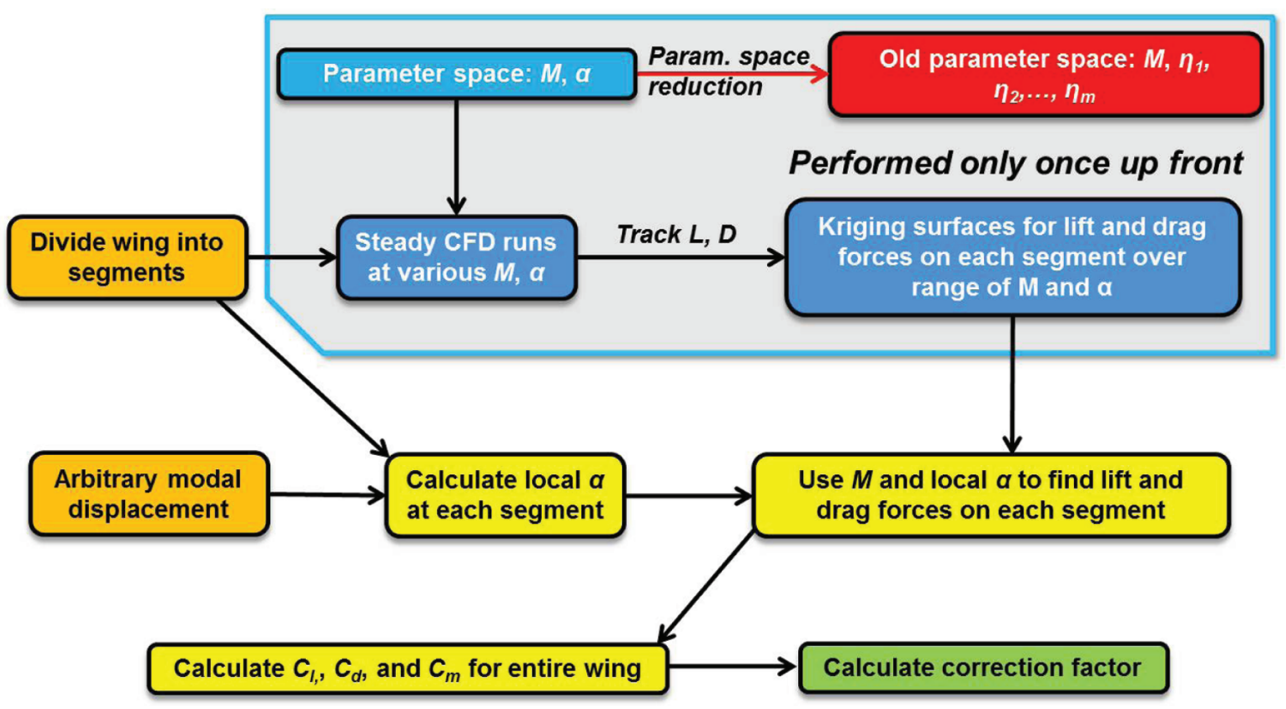

Fig. 5 Method-of-segments process. 
Table 1 ROM variations

\begin{tabular}{ccl}
\hline \hline ROM & $f_{c}$ calculation & Comment \\
\hline A & MoS & Direct calculation of $f_{c}$ at each time step using MoS \\
B & MoS/kriging & Points placed at location of maximum surface error \\
C & CFD/kriging & Mostly the same sampling points as ROM $B$ \\
D & N/A & Linear ROM $y_{\text {conv }}$ \\
\hline \hline
\end{tabular}

ROM B, is created by first generating an initial number of sampling points in the Mach number-modal amplitude parameter space via Latin hypercube sampling. Using MoS, the correction factor at each of these points is found, and a kriging surface is computed. Next, the sampling point procedure from [1] is implemented by finding the location of the surface's maximum error through the use of the builtin MATLAB predictor function [22]. Then, an additional point is placed at this location, the surface is recomputed, and the maximum error is recalculated. The whole process is repeated until the stopping criterion is met. ROM A can be thought of as the limit of ROM B if infinite sampling points are used. Figures 6 and 7 show schematics highlighting the differences between ROMs A and $\mathrm{B}$.

The next ROM considered here, ROM C, is calculated using the same sampling points as in ROM B, but the correction factor values at each point are computed using individual direct CFD simulations rather than MoS. Its schematic is shown in Fig. 8 and differs from that of ROM B by replacing MoS block with CFD simulations. Note that, in actuality, ROM C consists of fewer sampling points than ROM B. This is due to CFD code limitations because some of the runs at higher modal deformations ran into numerical issues and thus did not produce results. Finally, ROM D consists of the linear ROM $y_{\text {conv }}$ found using linear convolution and superposition.

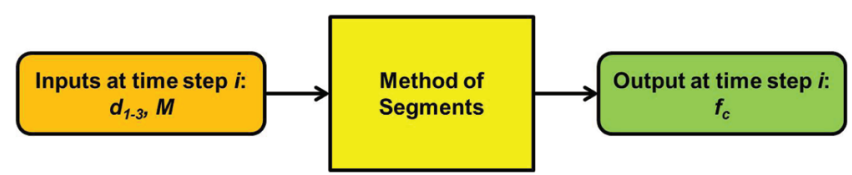

Fig. 6 Schematic for ROM A.

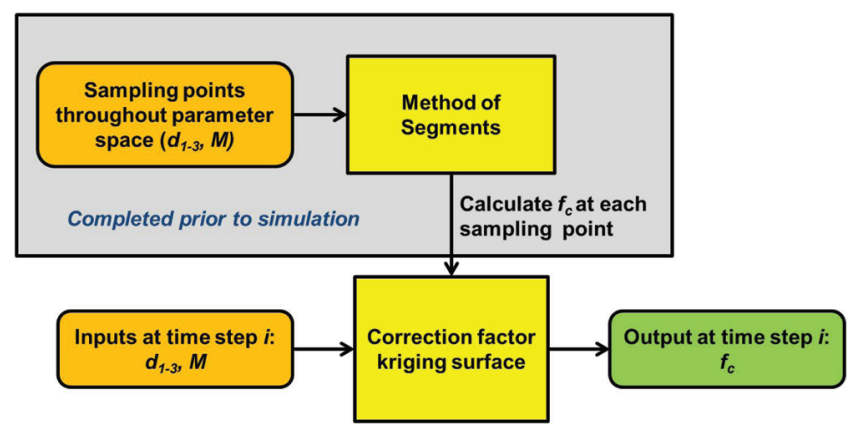

Fig. 7 Schematic for ROM B.

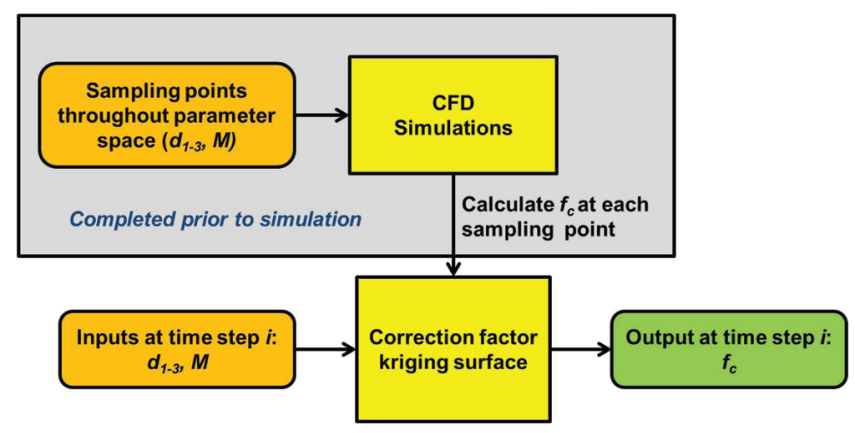

Fig. 8 Schematic for ROM C.

\section{F. Selection of Step Response}

One final item that needs to be discussed is the selection of the step response to use for $y_{\text {conv }}$ when calculating the ROM response. In reality, one will not have an unlimited number of step responses to choose from; thus, in general, the Mach number of the simulation, $M_{\text {sim }}$, will not be equal to the Mach number of the step response, $M_{\text {step }}$. The results sections provide several studies of this problem, investigating ROM errors as $M_{\text {sim }}$ moves away from $M_{\text {step }}$. Two methods have been developed for finding the best final ROM response in the presence of multiple $M_{\text {step }}$ values, shown graphically in Fig. 9. Method 1 uses a weighted average of the two ROM responses computed using the next higher and next lower $M_{\text {step }}$ values. Method 2 simply uses the ROM response computed from the closest value of $M_{\text {step }}$ to that particular value of $M_{\text {sim }}$. Note that, for cases where $M_{\text {step }}=M_{\text {sim }}$, the methods are identical. Figures 10 and 11 show block diagrams for methods 1 and 2, respectively, outlining how they would be implemented in a full simulation framework. For method 1, the weighted average of two ROM responses is passed back to the simulation framework, whereas for method 2, the response from the closest $M_{\text {step }}$ is passed back. Note that among the main differences in the methods is the fact that two ROM computations are necessary for method 1 because individual ROM responses must be calculated at each of two $M_{\text {step }}$ values, whereas only one such calculation is necessary for method 2 .

\section{Numerical Studies Overview}

The ROM methodology in the subsonic through supersonic regimes is assessed by comparing ROM results with full-order CFD simulations consisting of prescribed sinusoidal modal oscillations of each mode of interest in a particular problem. To begin with, specific focus is given to the Mach number range from 0.9 to 1.1 , which encompasses the transonic regime near Mach 1. For cases near Mach 1, mixed sub- and supersonic flow is present, which adds nonlinearities to the flowfield. Then, the Mach range is zoomed out to encompass separate subsonic and supersonic Mach ranges, from Mach 0.3 to 0.9 and 1.1 to 3.0, respectively. The final set of results shows a more practical application of the ROM, investigating the ROM's errors over a single test case from Mach 0.3 up through 3.0, including the transonic regime. Finally, a discussion about ROM computational cost is included. Before the results are shown and discussed, however, the geometry, CFD solver, and error metrics used in this work are described.

\section{A. AGARD 445.6 Wing}

The geometry upon which these test cases are performed is the AGARD 445.6 wing [24], which has been used in wind-tunnel aeroelastic tests as well as for computational aeroelastic studies [20,25-28]. A structured CFD grid has been obtained from NASA

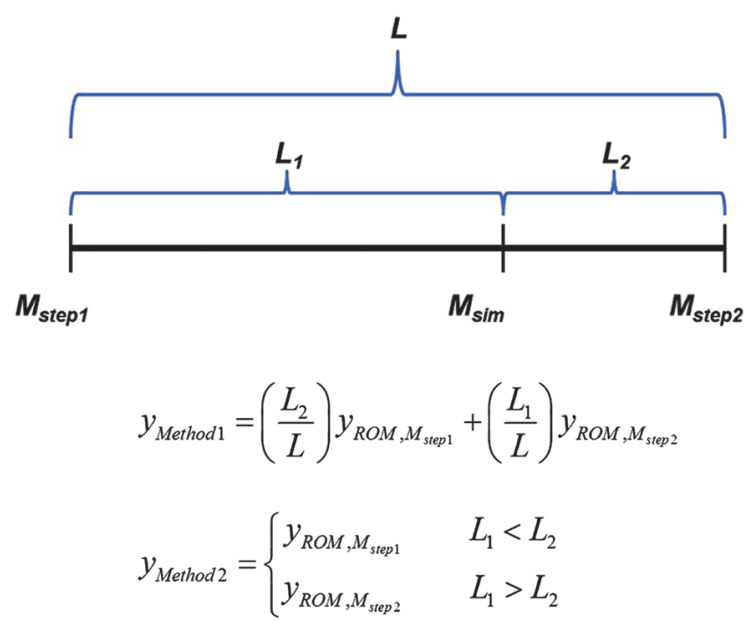

Fig. 9 Diagram for ROM calculation methods. 


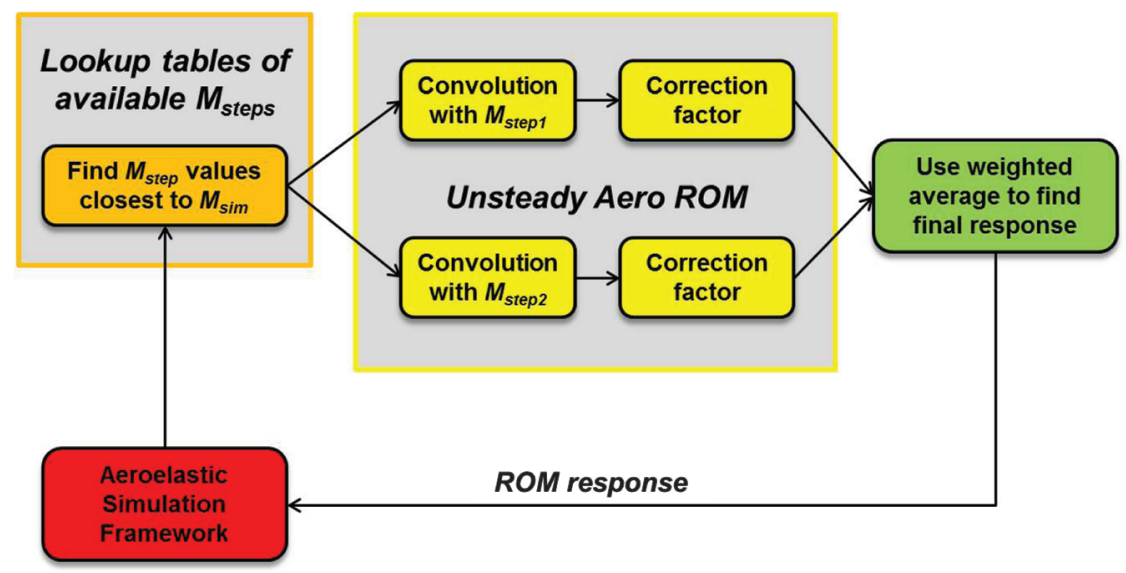

Fig. 10 Block diagram for method 1 implementation.

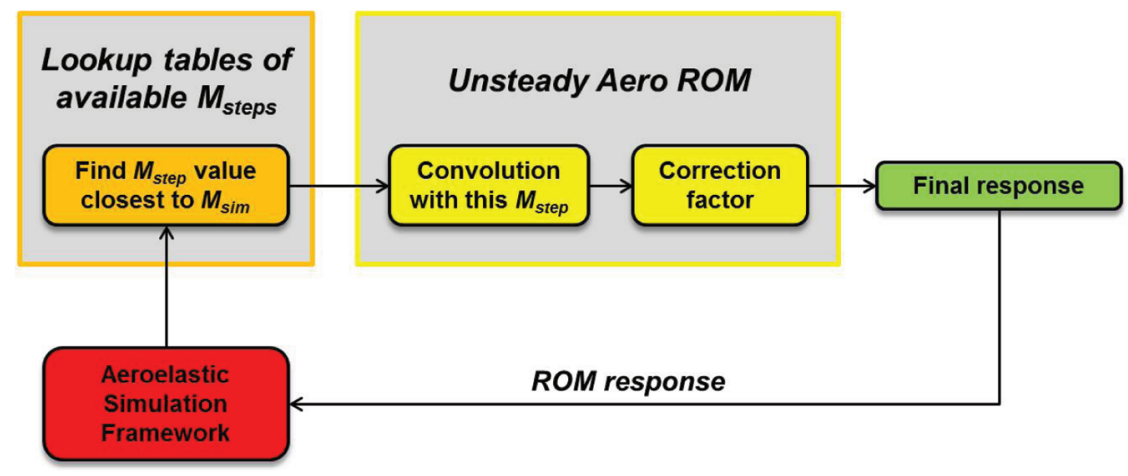

Fig. 11 Block diagram for method 2 implementation.

Langley Research Center and has dimensions of $65 \times 193 \times 41$, with the $i$ direction being along the span, $j$ direction along the chord, and $k$ direction normal to the wing surface. Figure 12 shows the grid as well as a zoomed-in figure of the wing itself. Oscillations of the first three elastic mode shapes of the wing are considered. These mode shapes, shown in Fig. 13, are the same that have been used in other studies as well $[29,30]$. Note that, for each mode shape, the unit step input corresponds to a maximum wing deflection of just around $0.1 \%$ of the span (3.5\% of the root thickness).

The AGARD 445.6 wing has a very thin cross-sectional geometry, with a maximum root thickness of about $4 \%$. Because of this, the onset of transonic effects, signaled by the presence of mixed sub- and supersonic flow, will be delayed until very close to Mach 1 in comparison with other, thicker airfoils. Evidence of mixed flow can be found by looking at the pressure contours over the wing because the transonic shock waves will cause pressure rises along the chord of the wing. Figure 14 shows the nondimensional pressure and Machnumber contours along the top half of the wing in steady flow at Mach 0.9889 and zero angle of attack; note that the pressure values of Fig. $14 \mathrm{a}$ are nondimensionalized in such a way that $p_{\infty}=1 / \gamma$, where $\gamma$ is the ratio of specific heats and equals 1.4. The presence of a shock on the airfoil can be seen from the sharp pressure increase in the chordwise direction along all spanwise stations of the wing, along with the corresponding decrease from supersonic to subsonic Mach

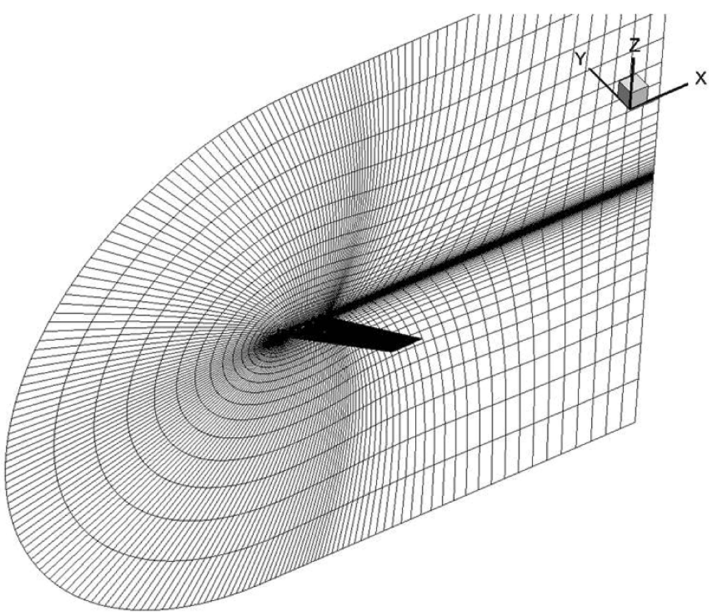

a) AGARD grid

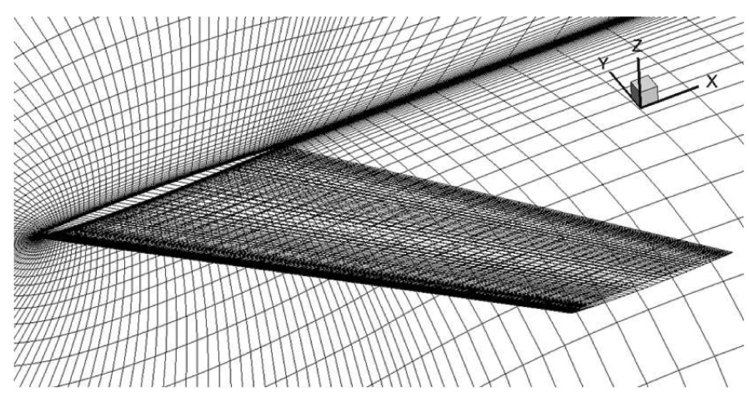

b) AGARD wing close-up 


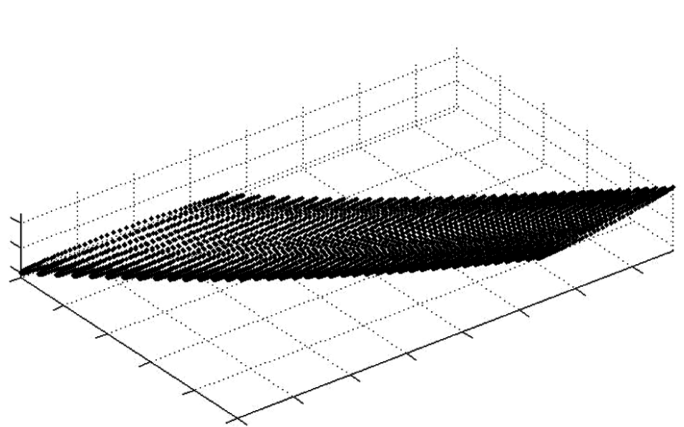

a) Mode $1, \omega_{1}=9.6 \mathrm{~Hz}$

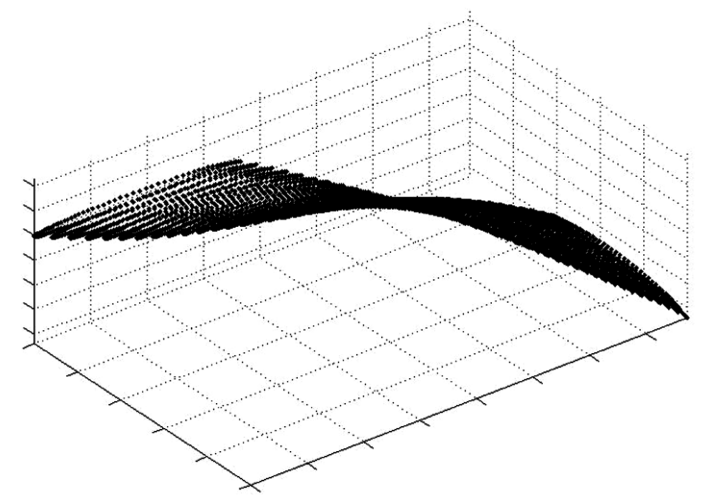

b) Mode 2, $\omega_{2}=38.2 \mathrm{~Hz}$

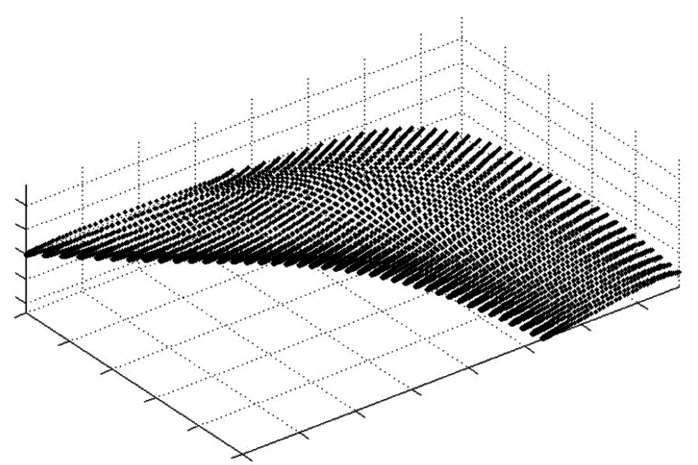

c) Mode 3, $\omega_{3}=48.2 \mathrm{~Hz}$

Fig. 13 AGARD 445.6 wing mode shapes.

numbers for this inviscid solution. To fully assess the ROM as the Mach number approaches 1 , results are obtained from simulations both from within this relatively narrow band of transonic effects, which are first seen typically for Mach numbers as low as around 0.98 and from a wider range of values from Mach 0.9 to 1.1 .

An additional note needs to be given regarding the performance of the ROM for situations with transonic flow and moving shocks. For other geometries, these transonic effects will be observed in a wider Mach range than the AGARD 445.6 wing, and so it is important to consider how these nonlinearities affect the ROM. Moving shocks create significant nonlinearities in localized regions of a wing. However, when these nonlinear pressure distributions are integrated over the entire wing to find the force and moment coefficients, the nonlinearities are softened to a degree. It is not the goal of the ROM to determine the exact shock location on the airfoil or the exact pressure distribution, rather the integrated force and moment quantities.

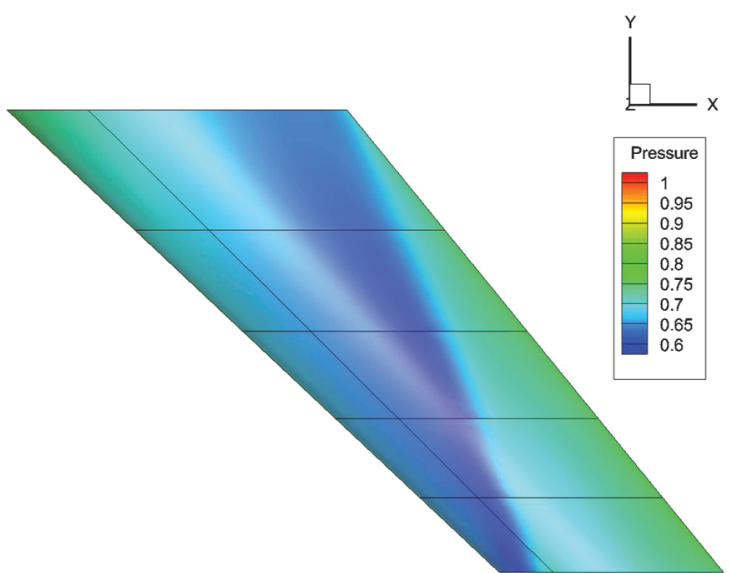

a) Pressure

\section{B. Computational-Fluid-Dynamics Reference Solutions}

The CFD code used in this study is CFL3Dv6, developed at NASA Langley [31]. The code is capable of solving the Euler/Navier-Stokes equations for both steady and unsteady flows on two- and threedimensional structured grids and has mesh-deformation capability. For the unsteady simulations, the CFD code inputs are modal deformations, in the form of step inputs or sinusoidal inputs, depending on the type of run being conducted. Grid velocities are derived from these modal inputs. For example, if a step input of amplitude $a$ is given to a particular mode at time step $n_{0}$ and $\Delta t$ is the time step being used, the grid velocities $\dot{\eta}$ are calculated as $[\underline{30,}, \underline{31}]$

$$
\dot{\eta}[n]=\left\{\begin{array}{ccc}
\frac{a}{\Delta t} & \text { for } & n=n_{0} \\
0 & \text { for } & n \neq n_{0}
\end{array}\right.
$$

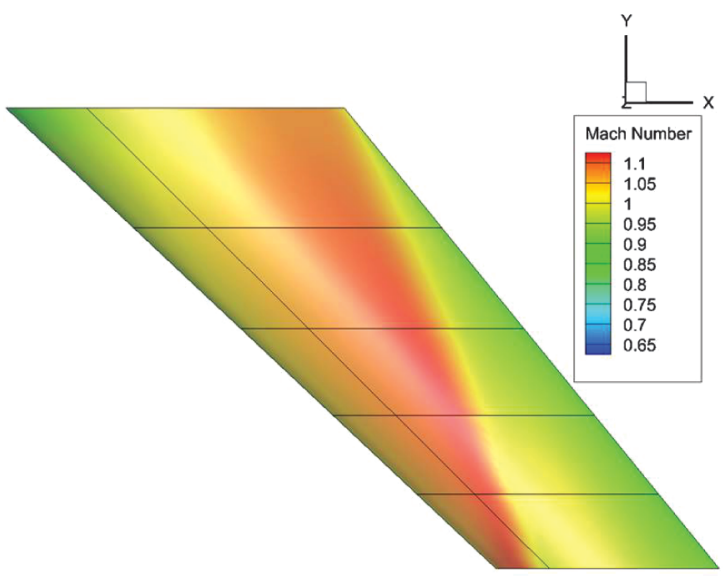

b) Mach number

Fig. 14 Contours on AGARD 445.6 wing, Mach $0.9889, \alpha=0$. 
Table 2 Method-of-segments testing parameters

\begin{tabular}{ccc}
\hline \hline Parameter & Minimum & Maximum \\
\hline$M$ & 0.9 & 1.1 \\
$d_{1}$ & -60 & 60 \\
$d_{2}$ & -40 & 40 \\
$d_{3}$ & -40 & 40 \\
\hline \hline
\end{tabular}

Table 3 Method-of-segments $L_{1}(\%)$ errors over 40 test cases (mean/standard deviation)

\begin{tabular}{lcc}
\hline \hline & Subsonic & Supersonic \\
\hline$C_{l}$ & $4.18 / 2.39$ & $4.23 / 3.27$ \\
$C_{d}$ & $5.60 / 5.20$ & $5.90 / 5.81$ \\
\hline \hline
\end{tabular}

The response quantities are the lift, drag, and moment coefficients, though the generalized aerodynamic forces (GAFs) could also be chosen. All of these quantities are directly output by the CFL3D code.

\section{Error Metric}

The error metric used to judge the accuracy of the ROM is the $L_{1}$ error. First, the mean absolute difference between the ROM and CFD results at each time step is found, and this difference is normalized by the range spanned by the CFD results. For a simulation over $N$ time steps, it yields

$$
L_{1} \text { error }=\frac{\frac{1}{N} \sum_{i=1}^{N}\left(\left|y_{\mathrm{ROM}, i}-y_{\mathrm{CFD}, i}\right|\right)}{\max \left(y_{\mathrm{CFD}}\right)-\min \left(y_{\mathrm{CFD}}\right)} \times 100 \%
$$

where $y_{\mathrm{ROM}, i}$ and $y_{\mathrm{CFD}, i}$ are the respective ROM and CFD response values found at time step $i$, and the denominator represents the difference between the maximum and minimum values found over all time steps of the ROM response.

\section{Method-of-Segments Testing}

The first set of results to present is a basic test of the accuracy of the MoS simplified model. For these tests, lift and drag coefficients found with MoS are compared with those found with direct CFD simulations for the same sample cases. A total of 40 test cases in each of the Mach number ranges from 0.9 to 1.0 (denoted the subsonic runs) and 1.0 to 1.1 (supersonic runs) is selected. The various parameter ranges for these sample cases are shown in Table 2. The MoS kriging surfaces themselves are computed using a total of 152 sampling points arranged in a lattice-type pattern in each of the suband supersonic portions of the overall Mach-number range considered here. The tip and root segment drag force kriging surfaces are shown in Fig. 15; the black dots represent the values found at each of the sampling points using CFD, corresponding to step 2 in the MoS procedure outlined previously. Table 3 shows the results of the comparison; the mean $L_{1}$ errors are under $5 \%$ for the lift coefficient in each of sub- and supersonic ranges, whereas the drag coefficient mean errors are under $6 \%$. These results demonstrate the potential of the method for use in error estimation and ROM correction factor sampling point determination.

\section{Results near Mach 1}

The first results presented are those for test cases near Mach 1, within the Mach range of 0.9-1.1. The first CFD test cases for ROM assessment here can be divided into several different sets. The first consists of single modal oscillation test cases and is subdivided into amplitude and frequency tests, in which all parameters except for amplitude and frequency, respectively, are held constant. A sense of how the ROM errors vary with increasing values of these two parameters is presented.

Next, the ROM is extended to multiple modes of oscillation within this same Mach regime. To construct the ROM, the sampling point determination methodology along with the MoS simplified model described previously are used here. Correction factor kriging surface sampling point coefficient values are obtained both through direct CFD computation as well as MoS and compared to one another to determine the accuracy of the simplified model and thus its applicability to the problem. Finally, full-order CFD solutions are compared to ROMs constructed with both MoS and CFD-calculated correction factor values.

\section{A. Single-Mode Tests}

The first set of investigations consist of characterizing the applicability of the ROM methodology to oscillations of the first mode only. The first items to consider are the ranges of parameters, including Mach number, oscillation amplitude, and oscillation frequency, to use during testing; these ranges are selected to be those shown in Table $\underline{4}$. The corresponding kriging surfaces, shown in Fig. 16, are constructed for the correction factors corresponding to each of the lift, drag, and moment coefficients. Because of the relatively large gradients around Mach 1, separate surfaces are

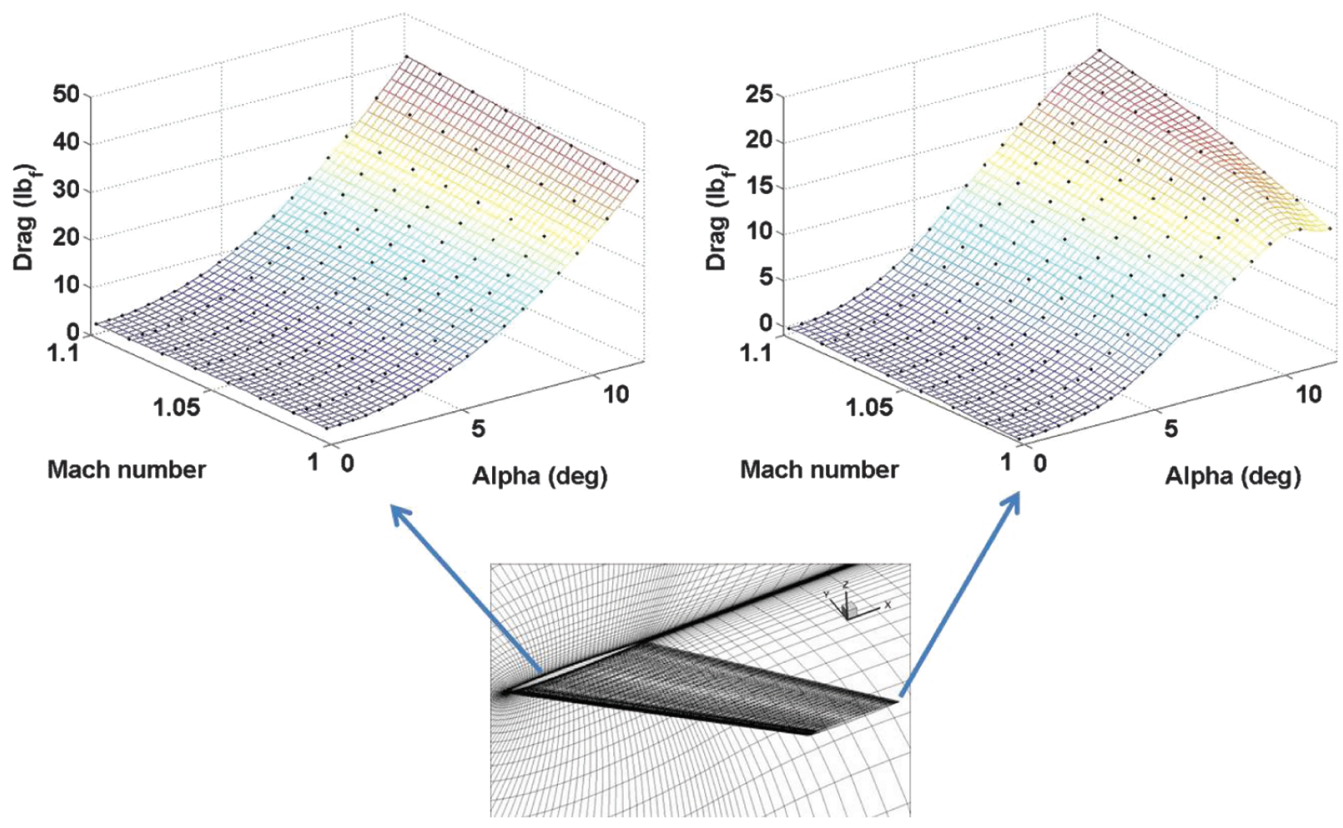

Fig. 15 Method-of-segments kriging surfaces, tip and root segments, Mach 1-1.1. 
Table 4 Single modal oscillation parameter values

\begin{tabular}{ccc}
\hline \hline Parameter & Minimum & Maximum \\
\hline$M$ & 0.8 & 1.2 \\
Amplitude & -100 & 100 \\
Frequency, $\mathrm{Hz}$ & 9.6 & 48 \\
\hline \hline
\end{tabular}

constructed for sub- and supersonic Mach numbers. Also, because the AGARD 445.6 airfoil is symmetric, negative amplitude lift and drag coefficient values are simply the opposite of and the the same as, respectively, those found for the corresponding positive amplitude, thus reducing the total number of CFD runs required. A total of 81 sampling points, found using Latin hypercube sampling of the parameter space, is used for the subsonic kriging surface, whereas 63 points are used for the supersonic surface. An offset value $\delta$ of $10^{6}$ is used. However, note that, in the plots of Fig. 16, smaller $\delta$ values are used as indicated; this is done to emphasize the difference of the correction factor values compared to unity.

\section{Amplitude Tests}

To test the accuracy of the ROM as the amplitude of oscillation increases, sinusoidal oscillation test cases are generated with constant Mach number and oscillation frequency but varying oscillation amplitude. These tests are repeated for both Mach 0.9 and Mach 1.1. For each test, two ROMs are constructed, one with the step input computed at Mach $0.9\left(M_{\text {step }}=0.9\right)$ and the other with a step input at Mach $1.1\left(M_{\text {step }}=1.1\right)$; the oscillation frequency is $\omega_{1}=9.6 \mathrm{~Hz}$. The results from each of the two ROMs for tests at Mach 0.9 (dashed lines) are compared to uncorrected ROM results (solid lines) in Fig. 17.

Figure 17 shows that, while the correction factor ROM shows improved agreement with the CFD results for the lift and moment coefficients, the overall errors seen for both the corrected and uncorrected ROMs are relatively small. Also, the expected result is observed in which the ROMs having equal step response $\left(M_{\text {step }}\right)$ and simulation $\left(M_{\text {sim }}\right)$ Mach numbers generally have lesser errors, though errors for all correction factor ROMs here are small, under 5\%. One interesting feature of Fig. $17 \mathrm{c}$ is the fact that the uncorrected ROM errors for $M_{\text {step }}=1.1$ decrease with oscillation amplitude. For a qualitative picture of this, consider Fig. 18, which shows the comparisons for the moment coefficient at amplitudes of 5 (Fig. 18a) and 100 (Fig. 18b). Initially, the step Mach number of 1.1 results in an overprediction of peak moment coefficient values at lower amplitudes. However, as the oscillation amplitudes increase, this overprediction lessens, resulting in lesser error values. Finally, looking at the drag coefficient results in Fig. 17b, the uncorrected ROM does not match well at all with the CFD solution data; to visualize these large errors, a qualitative comparison of the drag ROMs at an amplitude of 100 is shown in Fig. 19, in which the uncorrected ROM peaks are significantly smaller than those from the corrected ROM and CFD results.

Figure 20 shows the results for the tests conducted at Mach 1.1. The overall trends and errors are similar to those seen at Mach 0.9, though rather than the moment coefficient, the lift coefficient shows a decrease in error with increasing amplitude for the uncorrected ROM results for $M_{\text {step }} \neq M_{\text {sim }}$.

\section{Frequency Tests}

To investigate the accuracy of the ROM as oscillation frequency increases, tests are conducted at constant Mach number and oscillation amplitude but with oscillation frequencies ranging from $\omega_{1}(9.6 \mathrm{~Hz})$ to $5 \omega_{1}$. As with the amplitude tests, these tests are repeated for both Mach 0.9 and Mach 1.1, and oscillation amplitude is held constant at 20 . These parameters result in reduced frequencies ranging from 0.06 to 0.29 for $M=0.9$ and 0.05 to 0.24 for $M=1.1$. Also, for each value of $M_{\text {sim }}$, results from ROMs constructed with $M_{\text {step }}=0.9$ and 1.1 are given. The results, displayed in Figs. 21 and 22 , show that the errors do increase with oscillation frequency. This is likely due to the increased unsteadiness inherent with increased reduced frequencies combined with a steady correction factor formulation. Also, for the most part, the ROM in cases where $M_{\text {step }}=M_{\text {sim }}$ performs better than the ROM in cases where $M_{\text {step }} \neq M_{\text {sim }}$. The only exception is the drag coefficient for the tests
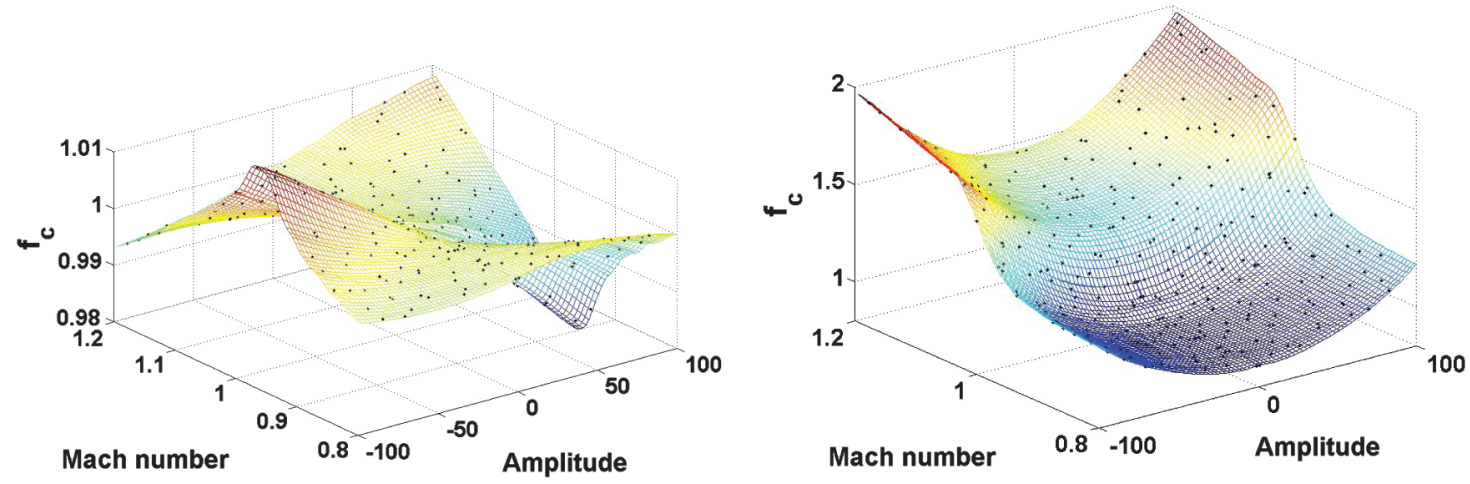

a) $f_{c}$, lift coefficient, $\delta=1$

b) $f_{c}$, drag coefficient, $\delta=0.01$

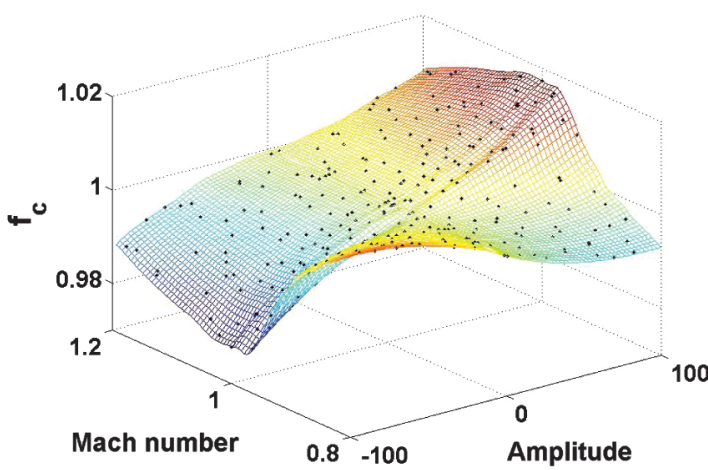

c) $f_{c}$, moment coefficient, $\delta=1$

Fig. 16 Kriging surfaces for mode 1. 

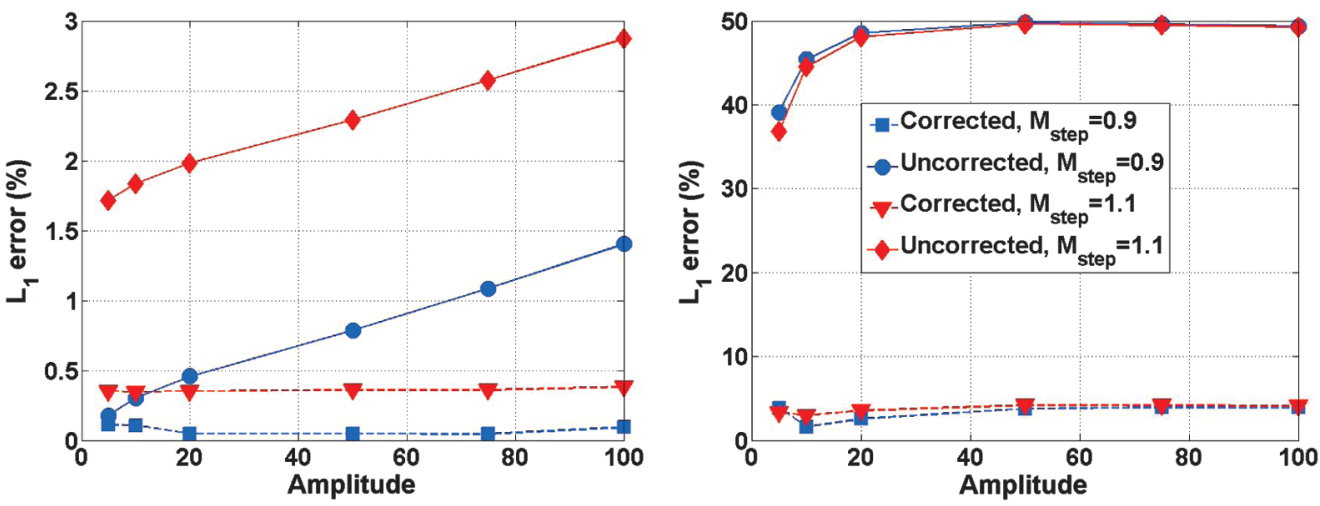

a) $C_{l}$

b) $C_{d}$

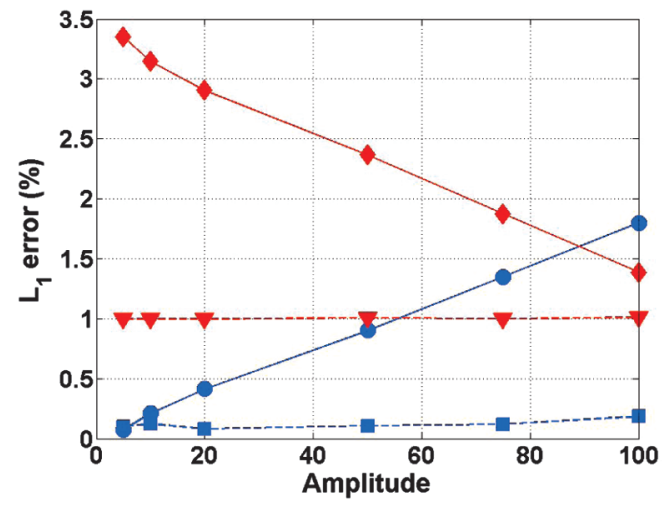

c) $C_{m}$

Fig. 17 Amplitude tests, Mach 0.9.

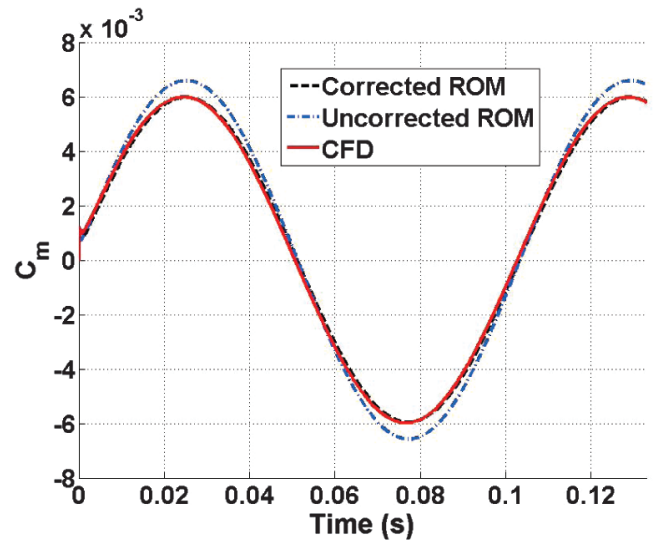

a) Amplitude 5

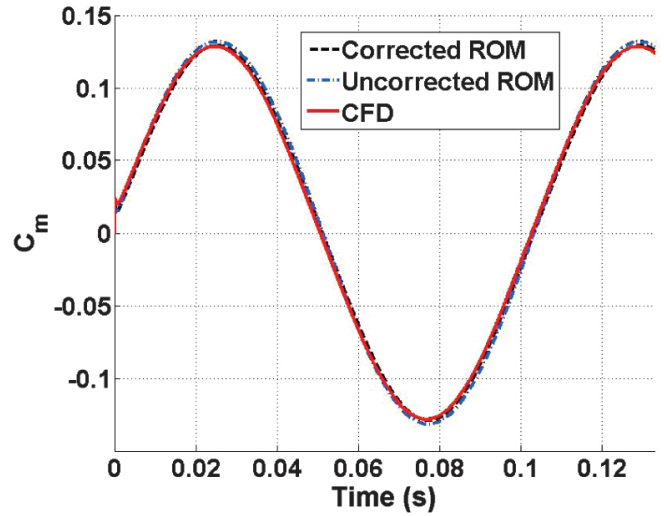

b) Amplitude 100

Fig. $18 C_{m}$ results, Mach 0.9, $M_{\text {step }}=1.1$.

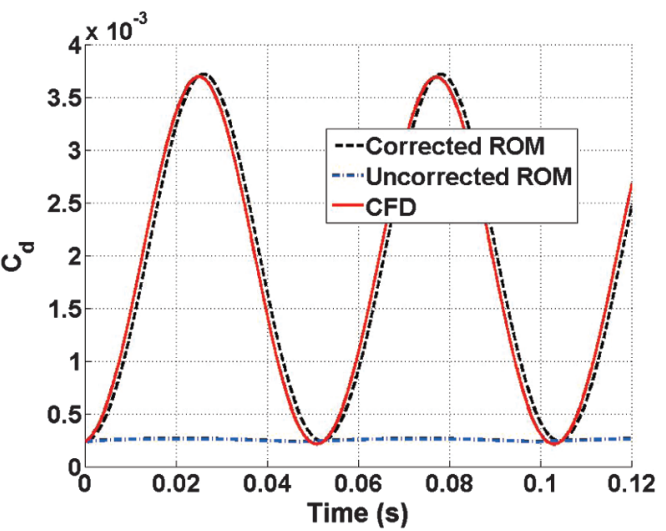

Fig. 19 ROM-CFD comparison, Mach 0.9, amplitude = 100, $M_{\text {step }}=0.9$. with $M_{\text {sim }}=1.1$, for which the ROM calculated with $M_{\text {step }}=0.9$ has slightly lower errors, though the errors for each ROM increase at a very similar rate.

For a more qualitative comparison, Fig. 23 shows the lift and drag comparisons for the test case corresponding to a frequency of $4 \omega_{1}$ and $M_{\text {sim }}=0.9$. For the lift coefficient, each of the two ROMs match well with the CFD results. For the drag coefficient, two sources of error can be seen. First, a slight amplitude discrepancy has developed. Second, a phase shift is observed between the ROM and CFD results.

To investigate this phase shift, the fast Fourier transform [32] is computed for both the ROM and CFD results for each set of runs shown in Table 5 , and the phase difference between the two responses is calculated. Figure 24 shows the lift, drag, and moment coefficient results for two separate series of results conducted at Mach 0.9 and Mach 1.1. Note that, for these tests, a single-mode ROM is used in which the correction factor is calculated through the use of a kriging 

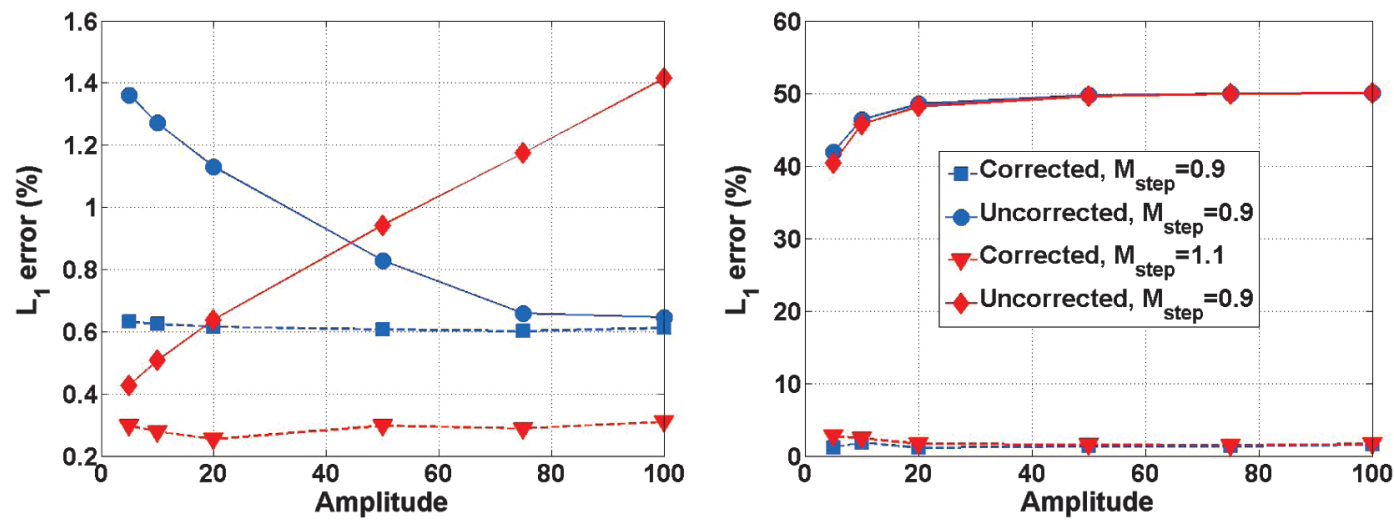

a) $C_{l}$

b) $C_{d}$

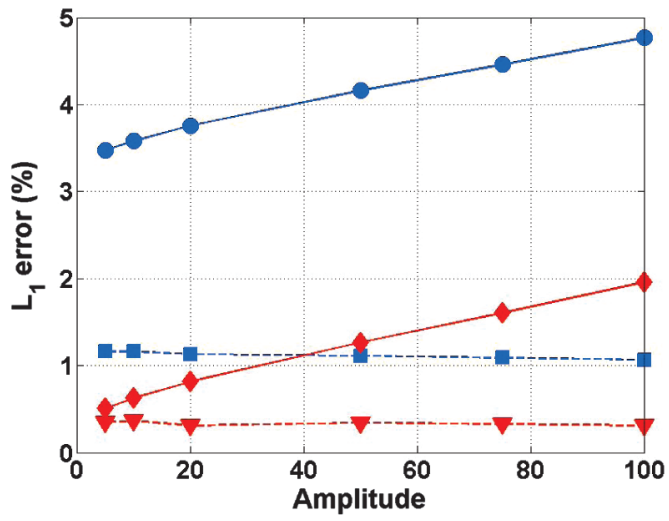

c) $C_{m}$

Fig. 20 Amplitude tests, Mach 1.1.
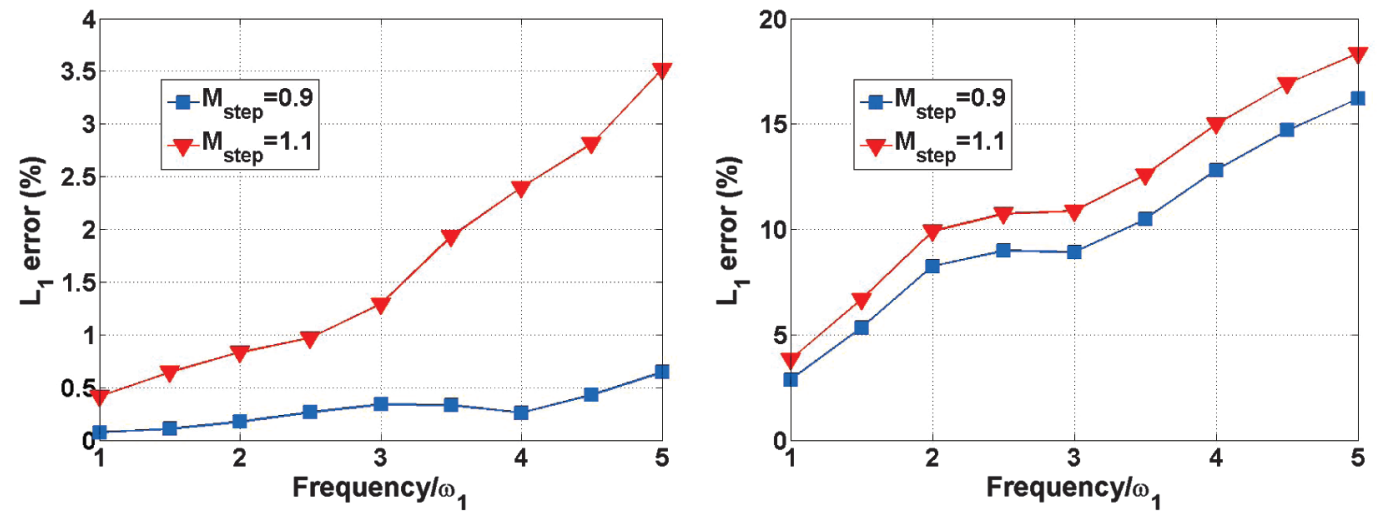

a) $C_{l}$

b) $C_{d}$

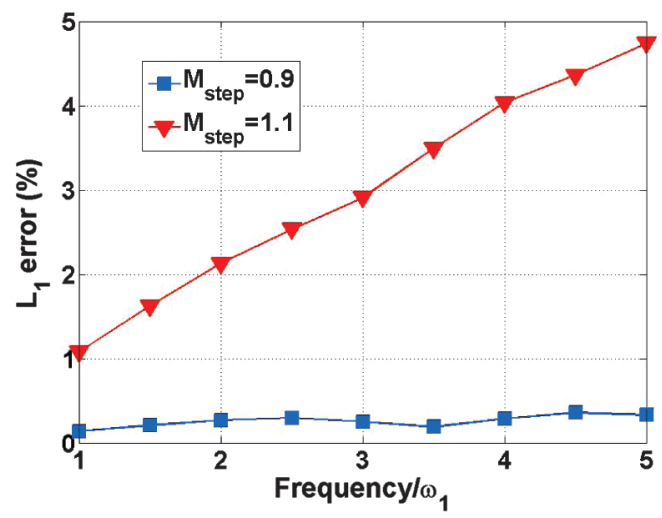

c) $C_{m}$

Fig. 21 Frequency tests, Mach 0.9. 

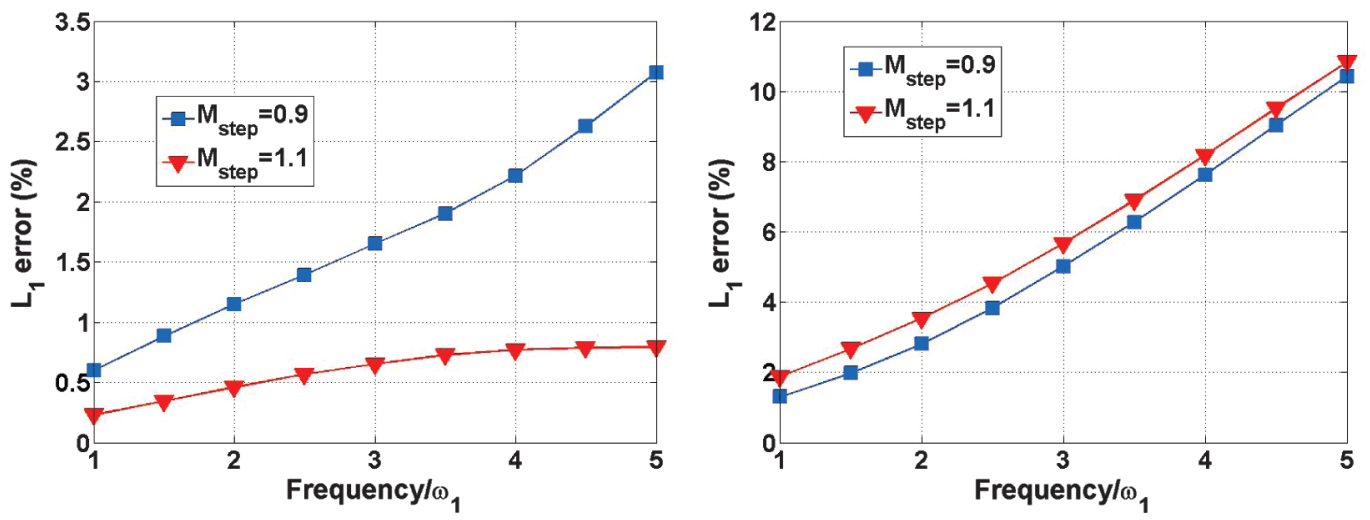

a) $C_{l}$

b) $C_{d}$

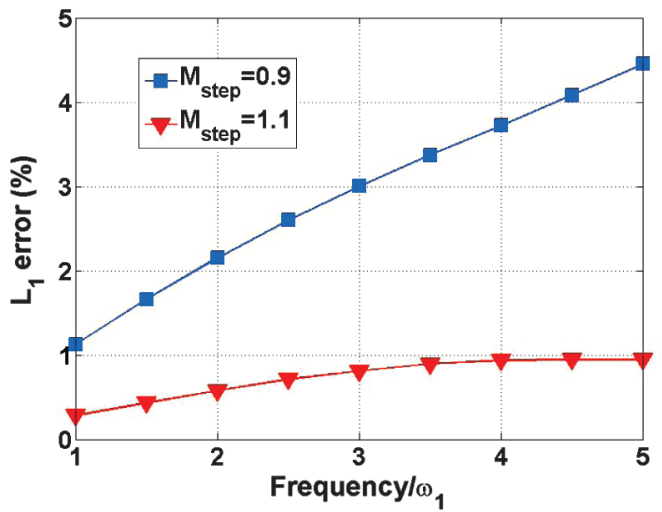

c) $C_{m}$

Fig. 22 Frequency tests, Mach 1.1.

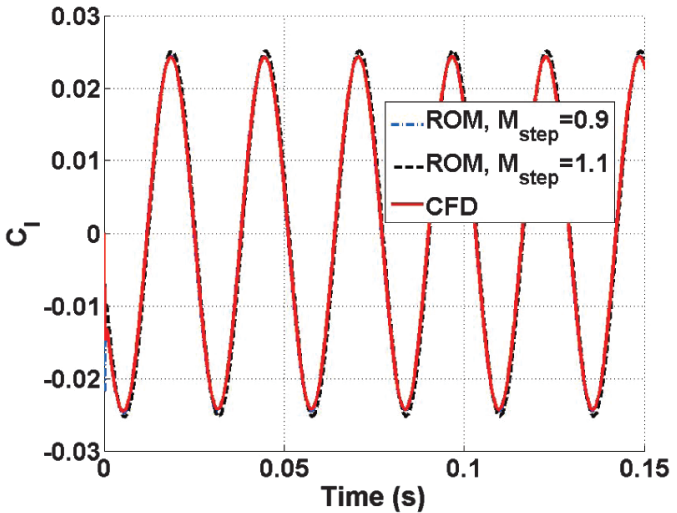

a) $C_{l}$

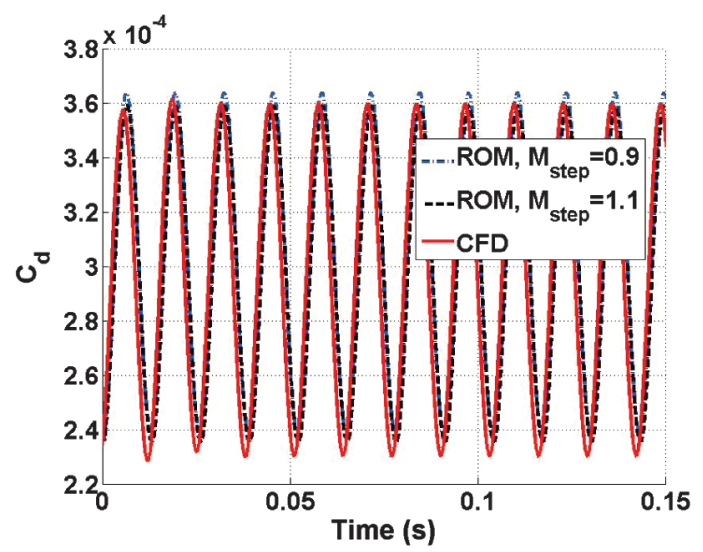

b) $C_{d}$

Fig. 23 ROM-CFD comparisons for $\omega=4 \omega_{1}$, Mach 0.9.

surface with sampling points computed using direct CFD simulations.

For each of the two sets of results, the drag coefficient shows a greater phase difference over the range of frequencies than the lift and moment coefficients, though this increase is more pronounced for the Mach 0.9 tests. This shift may be the result of a slight ROM-CFD offset in time that is being manifested as an increasing phase

Table 5 ROM phase shift test parameters

\begin{tabular}{ccccccc}
\hline \hline Test & $M$ & $M_{\text {step }}$ & $d_{1}$ & $\omega_{\min }, \mathrm{rad} / \mathrm{s}$ & $\omega_{\max }, \mathrm{rad} / \mathrm{s}$ & $k$ range \\
\hline 1 & 0.9 & 0.9 & 20 & 60.3 & $5 \omega_{\min }=301$ & $0.06-0.29$ \\
2 & 1.1 & 1.1 & 20 & 60.3 & $5 \omega_{\min }=301$ & $0.05-0.24$ \\
\hline \hline
\end{tabular}

difference with increasing oscillation frequency. Because of this overall phase difference increase with frequency, when looking at a specific test case, it is recommended to run a sample simulation at the highest frequency expected to be encountered and evaluate how the error fits in with the error tolerances for the problem.

\section{Single-Mode Tests in Mixed Flow}

The final item to discuss regarding the single-mode results is the specific performance of the ROM in cases with mixed flow. To investigate this, additional amplitude and frequency tests are conducted for Mach 0.99; the other parameters for the tests are the same as those mentioned previously. Figures 25 and 26 show the amplitude and frequency test results, respectively, for this study; note that $M_{\text {step }}=0.99$ for these cases as well. Similar trends are seen here as 


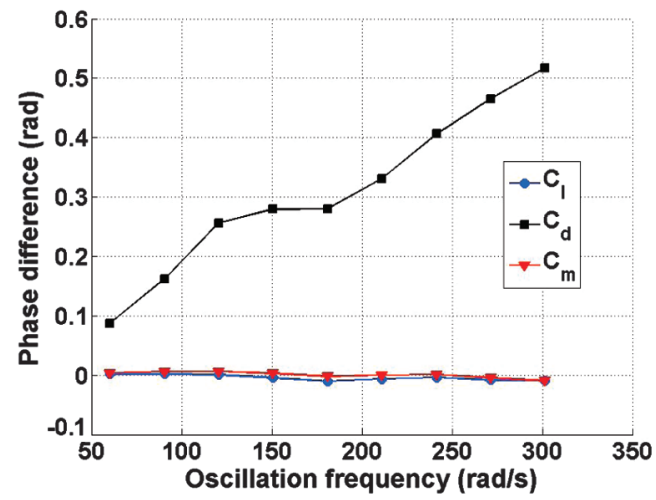

a) Mach 0.9

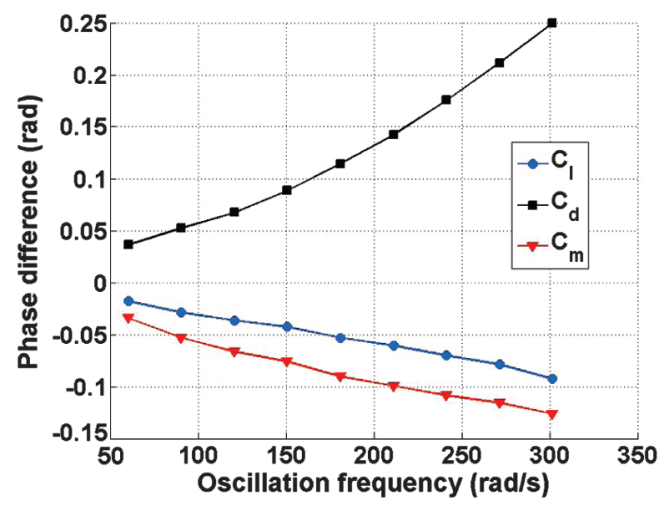

b) Mach 1.1

Fig. 24 ROM-CFD phase shift.

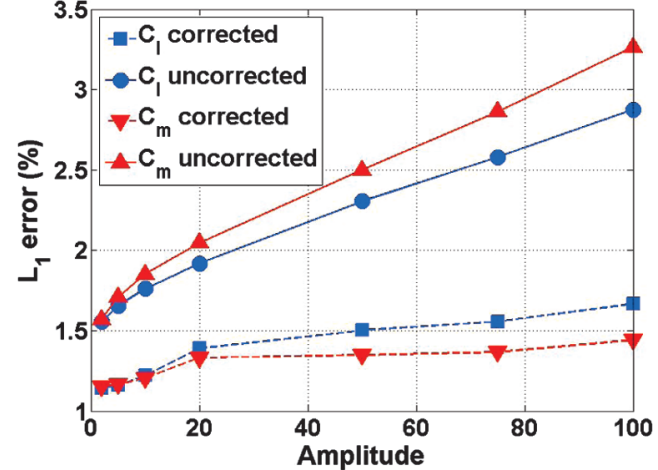

a) $C_{l}$ and $C_{m}$

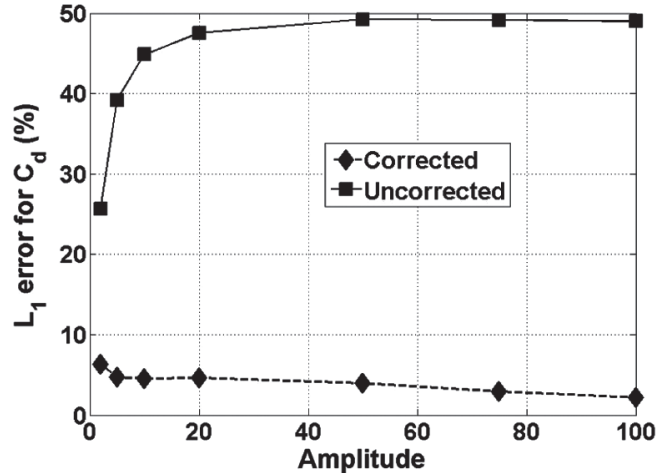

b) $C_{d}$

Fig. 25 Amplitude test, Mach 0.99.

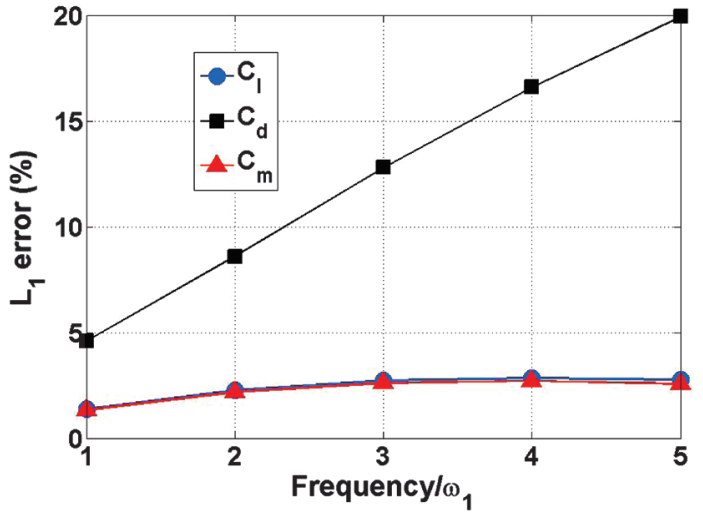

Fig. 26 Frequency test, Mach 0.99. compared to the amplitude and frequency tests conducted at Mach 0.9 and 1.1. The corrected ROM errors remain small over the entire range of amplitudes, whereas the uncorrected drag ROM results have the largest errors by far. For the frequency tests, lift and moment coefficient errors remain small, whereas the drag errors increase. To obtain a more qualitative sense of the errors, consider Fig. 27, which shows the ROM-CFD comparisons for the test cases corresponding to the nondimensionalized frequencies of 1 and 4 in Fig. 26. In addition to the slight phase shift as observed in previous frequency tests, an amplitude discrepancy develops as well for increased oscillation frequencies. However, the addition of extra kriging surface sampling points near the maximum modal amplitude in this run of 20 would likely help remedy this discrepancy. Overall, the single-mode tests in the mixed-flow transonic regime show similar results to the other Mach numbers considered.

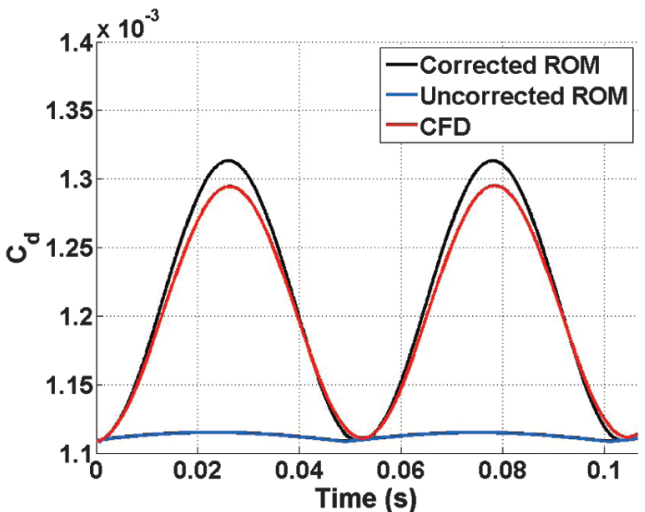

a) Frequency $/ \omega_{1}=1$

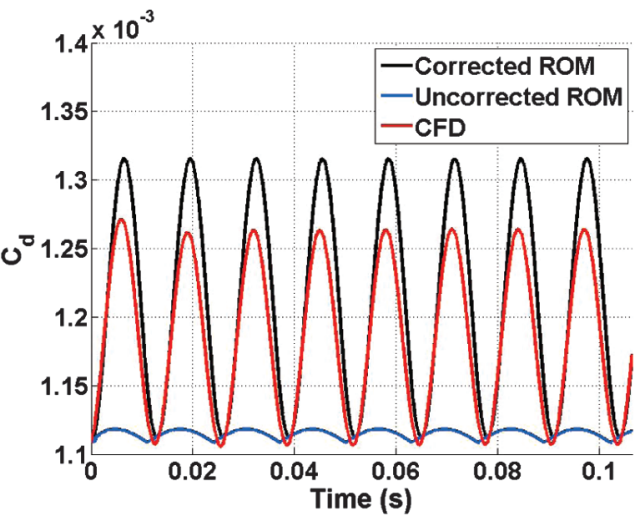

b) Frequency $/ \omega_{1}=4$

Fig. 27 ROM-CFD comparisons, frequency test cases, Mach 0.99. 
Table 6 ROM kriging surface sampling points

\begin{tabular}{ccc}
\hline \hline & \multicolumn{2}{c}{ Sampling points } \\
\cline { 2 - 3 } ROM & Subsonic & Supersonic \\
\hline A & N/A & N/A \\
B & $1011 / 1011$ & 1011 \\
C & $990 / 992$ & 992 \\
D & N/A & N/A \\
\hline \hline
\end{tabular}

Table 7 Parameter ranges for sinusoidal test cases, AGARD 445.6 wing

\begin{tabular}{lcc}
\hline \hline Parameter & Minimum & Maximum \\
\hline$M$ & 0.9 & 1.1 \\
$d_{1}$ & -50 & 50 \\
$d_{2}$ & -35 & 35 \\
$d_{3} \mathrm{rad} / \mathrm{s}$ & -30 & 30 \\
$\omega$ & 60.3 & 350 \\
$k$ & 0.05 & 0.29 \\
\hline \hline
\end{tabular}

\section{B. Multimodal Tests}

To investigate the ROM's applicability to multiple modes of oscillation, the first three modes of oscillation of the AGARD 445.6 wing are considered. MoS, described previously, has been selected as the simplified model to use to assist in ROM construction.

\section{Multimodal Oscillation Results}

The goals of applying the ROM methodology to multiple modes of oscillation of the AGARD wing are twofold. The first goal is to characterize the overall errors seen from the ROM, as has been the goal of the other sets of results discussed here. The second goal is to investigate the accuracy of a ROM constructed with the correction factor values calculated using MoS versus a ROM constructed with those same values computed with full CFD simulations. The preceding section demonstrated the potential for the method to calculate individual coefficient values, but now the challenge is to demonstrate whether or not it can be applied to the full ROM. With these goals in mind, the ROMs listed in Table 1 are all computed for these tests; Table $\underline{6}$ lists the number of correction factor kriging surface sampling points used for each ROM. Note that each ROM is constructed twice, once for Mach numbers less than 1 (Mach 0.9-1) and once for Mach numbers greater than 1 (Mach 1-1.1). All correction factor ROMs tested here use $\delta=100$.

Results obtained from these four ROMs are compared to a total of 100 full-order CFD simulations with sinusoidal oscillations of the first three modes of the AGARD 445.6 wing; half of these cases are in the subsonic portion of the Mach regime (Mach 0.9-1), and half are in the supersonic portion (Mach 1-1.1). For the subsonic test cases, $M_{\text {step }}=0.9$ is used for ROM construction, whereas $M_{\text {step }}=1.05$ is used for the supersonic cases. Table 7 highlights the ranges of the various parameters used for these tests. Note that the frequency range is chosen such that the minimum value corresponds to $\omega_{1}=$ $60.3 \mathrm{rad} / \mathrm{s}$, and the maximum value corresponds to slightly over $5 \omega_{1}$.

Figure 28 shows both the $L_{1}$ and $L_{\infty}$ drag coefficient error results for each of the four ROMs listed in Table 1 . The bars show the mean value of each of the error metrics over all $\overline{10} 00$ runs, whereas the error bars show the standard deviations.

For the two MoS ROMs, ROM A shows a slight improvement over ROM B for each of the error metrics, decreasing the mean $L_{1}$ error from around 11.5 to $9.8 \%$ and the $L_{\infty}$ error from around $30 \%$ to just over $27 \%$. This is to be expected due to the fact that ROM A calculates the MoS correction factor value at each time step, whereas ROM B obtains the correction factor value from a previously constructed kriging surface. Next, ROM D clearly performs the worst, with $L_{1}$ and $L_{\infty}$ errors of around 32 and $99 \%$, respectively. This demonstrates that the linear ROM is not suitable to model the drag coefficient. Finally, ROM C performs the best in terms of each of the error metrics when compared with the linear and MoS-based ROMs. For a better illustration of these comparisons, the ROM and CFD results for a number of specific test cases are shown in Figs. 29 and 30; the parameters and errors for these runs are listed in Table 8 .

In general, graphically speaking, two main sources of error can be seen. Figure 29a displays the results for test 1, which has the highest $L_{1}$ error for $\mathrm{ROM} C$ over all runs. Qualitatively, relatively large discrepancies can be seen between each of the ROMs and the CFD results. However, when looking at the total range spanned by the drag coefficient response value, it is relatively small. This is further illustrated in Fig. 29b, which shows the ROM-CFD comparisons for test 2, which has some of the smallest error values out of all test cases. In addition to the ROMs for that test case, the values from test 1 are superimposed on the plot with the green lines. As can be seen, though the errors for test 1 are larger than those for test 2 , the range spanned by the response of test 2 is much larger. This shows that some of the large error values are due to small ranges spanned by the response quantity, resulting in small denominators in the error metric equation [Eq. (10)] and hence larger error values. Note that, for test 1 , the unexpected result of ROM A having a larger error than ROM B is observed. This appears to be the result of a slight constant offset introduced by ROM A for this particular case that has been magnified due to the small ranges of coefficient values spanned in the simulation.

For the second source of error, consider Fig. 30, which shows test cases having error values right around the mean. In these cases, the largest source of error appears to be amplitude discrepancies between the ROM and CFD results. In many situations, the predictions from ROM C tend to overpredict peak drag coefficient values, resulting in some error, whereas the peak comparisons for the MoS-based ROMs vary.

In addition to the drag coefficient, lift coefficient results for these same test cases are found as well, and the mean errors and standard deviations can be found in Fig. 31. These results are strikingly different from those found for the drag coefficient and show two main points. The first is that the errors for each of the ROMs are relatively
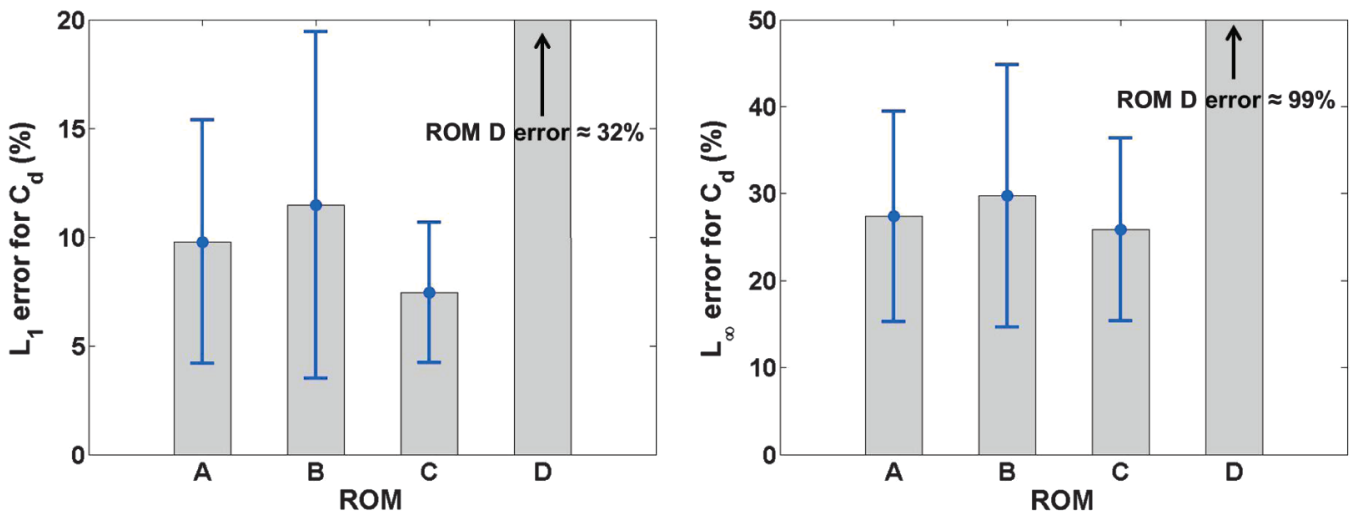

a) $L_{1}$

b) $L_{\infty}$

Fig. $28 C_{d}$ error results over 100 test cases. 


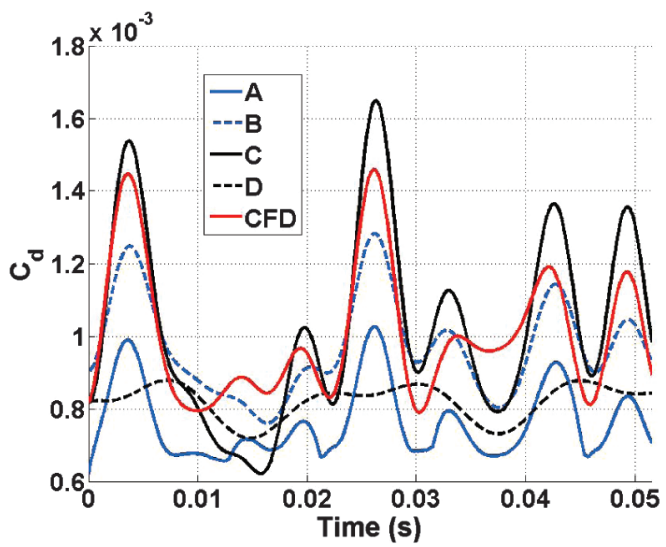

a) Test 1

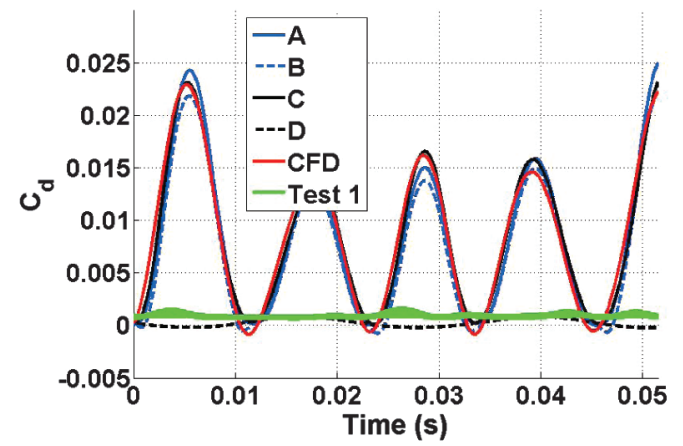

b) Test 2

Fig. 29 Example test cases, large and small $C_{d}$ errors.

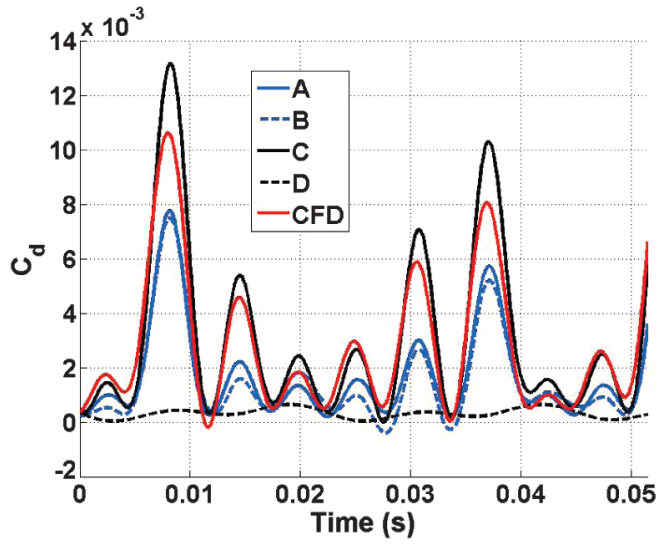

a) Test 3

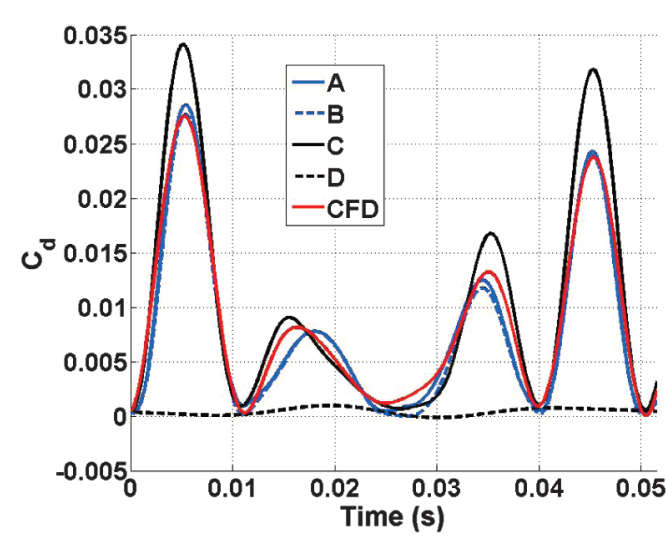

b) Test 4

Fig. 30 Example test cases, mean $C_{d}$ errors.

small, under $6 \%$ for the mean $L_{1}$ error for ROM C. The second is that the best agreement is found between ROM D (linear) and the CFD results, and each of the correction factor ROMs give slightly higher errors. This suggests that, for these test cases, the linear ROM is sufficient to model the lift response. The higher errors for the nonlinear correction factor ROMs (A-C) likely result from the fact that the addition of the nonlinear correction factor inherently results in some approximation errors. When looking at quantities like the drag coefficient, which are very nonlinear, the application of the correction factor, despite these approximation errors, still greatly improves the results and shows a significant decrease in error over the linear ROM. However, when the system itself can be well modeled as linear, these approximation errors in the application of the nonlinear correction factor result in a slight error overhead, thus increasing the errors in this case over the linear model. Figure 32 shows the ROMCFD comparisons for test cases 3 and 4 from Table 8, in which the improvement of results with the purely linear ROM over the nonlinear ROMs can be seen. For ROM C, the introduction of the correction factor generally results in a slight overprediction of peak lift coefficient values, resulting in larger error values. The results for the pitching moment are found to be similar in nature to the lift coefficient results in that ROM D is a good predictor of the response.

\section{Mixed-Flow Results}

As before with the single-mode tests, an important item to evaluate is the ROM's performance for multimodal oscillations in the presence of mixed transonic flow. To do so, consider the specific test cases out of the 100 multimodal oscillation test cases that are in the Mach 0.98 1 mixed-flow transonic regime for this wing. In all, 10 cases fall into that range. The mean $L_{1}$ errors and error standard deviations of these cases for each coefficient are shown in Table 9. The results show that the mean drag error for these specific cases is slightly higher than for all of the cases, whereas the lift mean error is comparable. To get a qualitative sense of where the errors are coming from, consider Fig. 33, which shows the ROM-CFD comparisons for the lift and drag coefficient for a sample case, dubbed here as case T1, having a drag error approximately that of the mean value for these mixed-flow cases; the parameters for this run are shown in Table 10. The main differences that can be seen between the ROM and $C \overline{F D}$ results for each coefficient are peak discrepancies. The ROM results overpredict

Table 8 Test case parameters and errors

\begin{tabular}{ccccccccccc}
\hline \hline & & & & & & & & \multicolumn{3}{c}{$C_{d}$ errors: $L_{1} / L_{\infty}$} \\
\cline { 7 - 10 } Test & $M$ & $d_{1}$ & $d_{2}$ & $d_{3}$ & $\omega_{1}, \mathrm{rad} / \mathrm{s}$ & $\omega_{2}, \mathrm{rad} / \mathrm{s}$ & $\omega_{3}, \mathrm{rad} / \mathrm{s}$ & ROM A & ROM B & ROM C \\
\hline 1 & 0.98 & -11.1 & 3.30 & -4.20 & 109 & 289 & 541 & $36.2 / 68.4$ & $12.7 / 30.3$ & $15.9 / 35.0$ \\
2 & 0.91 & 25.2 & -29.2 & 5.80 & 158 & 274 & 492 & $5.11 / 17.3$ & $6.26 / 22.9$ & $3.63 / 12.1$ \\
3 & 0.94 & 28.4 & -9.00 & -22.4 & 137 & 269 & 557 & $9.90 / 29.1$ & $12.0 / 30.9$ & $6.14 / 25.8$ \\
4 & 0.96 & 13.4 & -23.7 & 21.3 & 180 & 245 & 379 & $3.77 / 14.0$ & $4.58 / 16.1$ & $6.92 / 29.3$ \\
\hline \hline
\end{tabular}




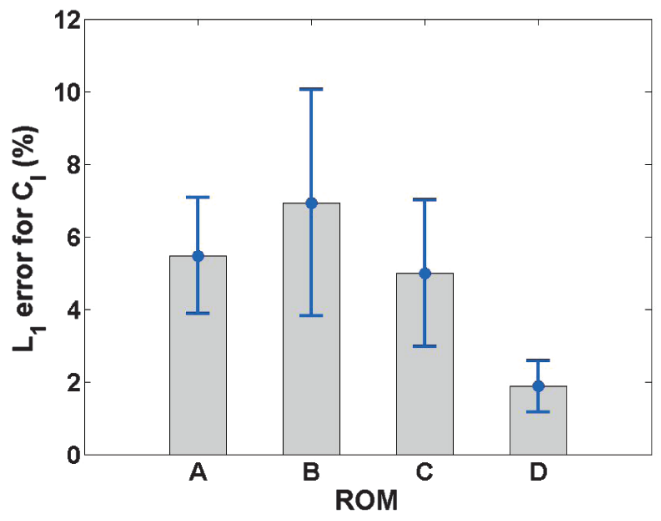

a) $L_{1}$

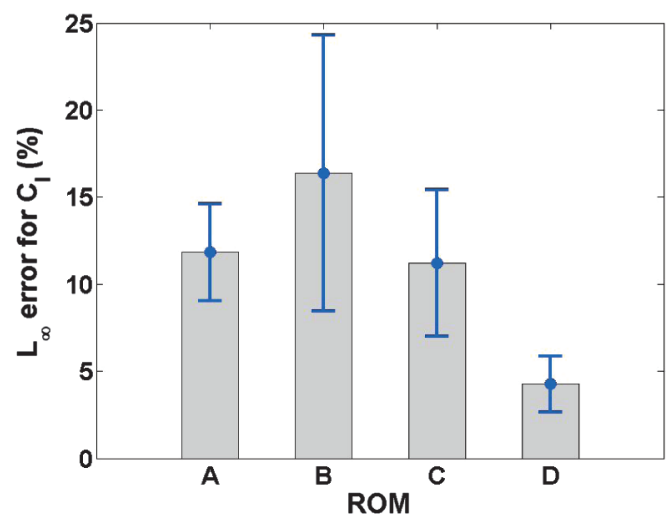

b) $L_{\infty}$

Fig. $31 C_{l}$ error results over 100 test cases.

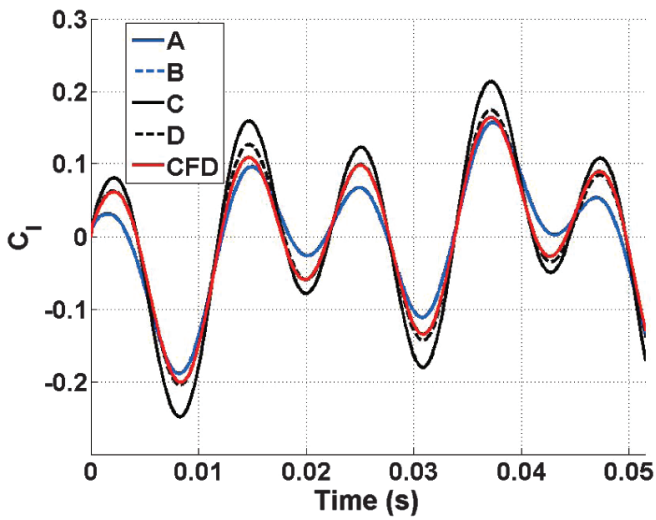

a) Test 3

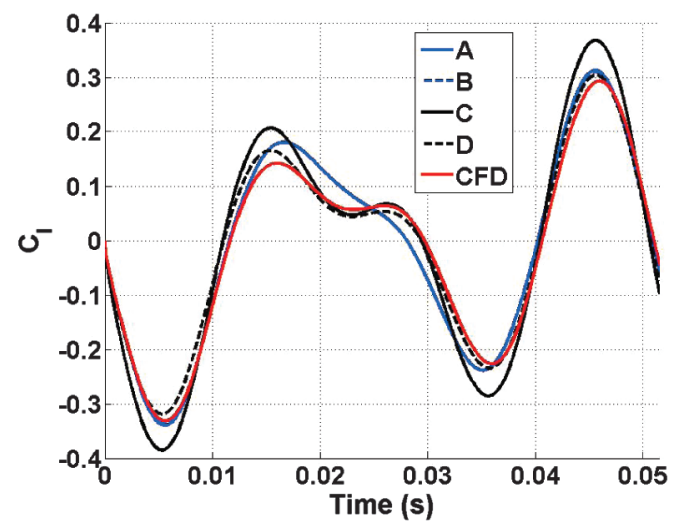

b) Test 4

Fig. 32 Example test cases, $C_{l}$ errors.

the peak amplitude values, resulting in some error for these test cases. Although the ROM results still show overall good agreement with the CFD solutions for the mixed-flow Mach range, a priori knowledge of the location of this range for a specific geometry would aid in identifying the regions within the parameter space where nonlinear transonic effects are prevalent. Additional correction factor sampling points may be required to reduce errors in those locations.

\section{Subsonic and Supersonic Results}

The next set of results zooms out from the narrow band of Mach numbers around Mach 1 and examines the ROM's applicability over a broader range of Mach numbers and Mach regimes. The AGARD 445.6 wing would be expected to fly up through the low supersonic Mach regime, and so the Mach numbers up to these low supersonic values comprise the parameter space considered here. In these results, two specific Mach number ranges are considered: Mach 0.3-0.9 (denoted the subsonic range) and Mach 1.1-3.0 (denoted the supersonic range). For these tests, as with the previous multimodal test cases, an offset $\delta$ value of 100 is used for ROM construction.

The test cases themselves for these results consist of defining 25 sets of modal parameters (both amplitudes and oscillation frequencies) and conducting simulations for all of those modal parameters at each one in a specific range of Mach numbers. The modal parameters are obtained through Latin hypercube sampling,

Table 9 Results for mixed-flow transonic cases

\begin{tabular}{ccc}
\hline \hline Coefficient & $L_{1}$ error, $\%$ & Standard deviation, $\%$ \\
\hline$C_{l}$ & 4.86 & 0.78 \\
$C_{d}$ & 9.78 & 2.41 \\
$C_{m}$ & 5.73 & 0.60 \\
\hline \hline
\end{tabular}

and the parameter space for these runs is displayed in Table 11. A number of different goals are accomplished through this analysis. First, these results allow for the error of the methodology in general to be analyzed as a function of Mach number, as the same modal parameters will be tested at different Mach numbers. This way, potential conclusions of the range of the ROM's applicability can be deduced. Next, the effect of separation between the simulation Mach number $M_{\text {sim }}$ and the step response Mach number $M_{\text {step }}$ will be examined. Third, the applicability of MoS to these Mach regimes will be studied. Finally the effect of $N_{\text {samp }}$ on the results will be quantified by comparing ROM errors with correction factor kriging surfaces constructed with anywhere from around 100 sampling points to just over 500 sampling points. Note that, except for the results specifically showing these $N_{\text {samp }}$ comparisons, the maximum number of $500+$ sampling points are used for the correction factor kriging surfaces. Also, the ROM results shown here are equivalent to ROM C from Sec. V, in which the correction factor values have been computed directly through individual CFD simulations.

\section{A. Drag Coefficient Results}

The first plot to consider is Fig. 34, which shows the mean drag coefficient errors over all cases in which the step response Mach number $M_{\text {step }}$ is equal to the simulation Mach number $M_{\text {sim. Note that }}$ each data point on the plot is the mean of the 25 sinusoidal test cases conducted at that particular Mach number, and the error bars represent one standard deviation.

Several items become apparent by looking at the plots. First, in the subsonic Mach range, the errors decrease significantly as Mach number increases, falling from just over $16 \%$ at Mach 0.3 to just under 5\% for Mach 0.9. Along with the mean values, the spread in error values decreases as well, a fact that can be seen by the reduction in standard deviation values. Next, after a slight decrease in error from the Mach 1.1 value, the supersonic Mach range errors are relatively 


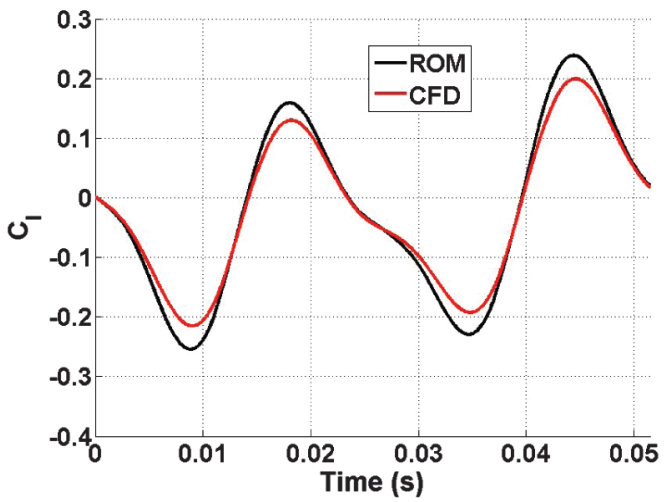

a) Lift comparison

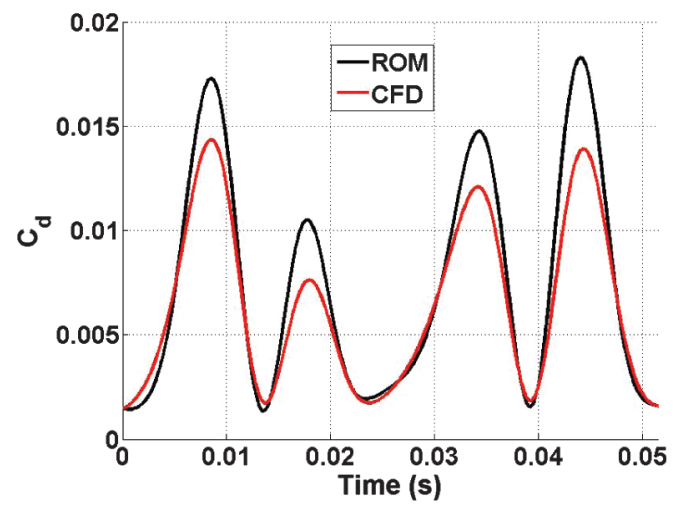

b) Drag comparison

Fig. 33 ROM-CFD comparisons for case T1.

constant, hovering at just around 3\%. These values are, in general, smaller than the subsonic values, again pointing to an increase in ROM accuracy with increasing Mach number.

In practice, the values for $M_{\text {sim }}$ in general would not be expected to be equal to $M_{\text {step }}$. Because of this, it is necessary to investigate the ROM errors for each of the simulations at a particular value of $M_{\text {sim }}$ that have been calculated using a range of $M_{\text {step }}$ values. Figure $\underline{35}$ displays the mean errors and standard deviations for each value of $M_{\text {sim }}$ for ROMs that have been calculated at each of the values of $M_{\text {step }}$. For example, consider the value $M_{\text {sim }}=0.5$. To calculate the corresponding data point in Fig. 35a, ROMs need to be computed for each of the 25 sinusoidal simulations conducted at $M_{\text {sim }}=0.5$ for $M_{\text {step }}=\left[\begin{array}{llll}0.3 & 0.5 & 0.7 & 0.9\end{array}\right]$, which gives a total of $25 \times 4=100$ ROM-CFD comparisons. The errors from these 100 comparisons are then used to compute the mean value and standard deviation data in the figure.

In general, the trends seen in Fig. 35 are similar to those observed in Fig. 34. The errors decrease with Mach number in the subsonic range, and the supersonic errors are smaller than the subsonic errors. A slight increase in error with Mach number is seen in the higher Mach numbers of the supersonic range, even climbing higher than the $M_{\text {sim }}=1.1$ error, but the overall error values remain small, with mean values under $6 \%$ in all cases.

The last item to visualize for the drag coefficient errors is how the errors change with $M_{\text {step }}$. Consider Fig. 36, which shows the errors for each value of $M_{\text {sim }}$ as a function of $\overline{M_{\text {step }}}$. Each data point in this plot is computed by calculating the mean error over all 25 runs conducted at a specified value of $M_{\text {sim }}$ using a constant value of $M_{\text {step }}$. For the subsonic Mach range shown in Fig. 36a, the errors remain fairly constant for each value of $M_{\text {sim }}$ over the range of $M_{\text {step }}$. Also, as seen previously, the errors decrease with Mach number, with all data points for $M_{\text {sim }}=0.9$ having the smallest error at each $M_{\text {step }}$ value and all data points at $M_{\text {sim }}=0.3$ having the largest.

However, the supersonic results in Fig. 36b show different trends. First, all values of $M_{\text {sim }}$ follow the same progression of decreasing error as $M_{\text {step }}$ increases except for $M_{\text {sim }}=1.1$, which remains fairly constant over the range. Next, the greatest value of $M_{\text {sim }}, 3.0$, has the largest errors in general over the Mach range, except for the two highest values of $M_{\text {step }}$ tested, where the constant value of the $M_{\text {sim }}=1.1$ error is the largest. In general, for a specific value of $M_{\text {step }}$, the error decreases as $M_{\text {sim }}$ is reduced, which is the opposite of what is seen in Fig. 36a. This result may be a function of how far apart $M_{\text {sim }}$ and $M_{\text {step }}$ become in some circumstances. The spread of Mach values in the supersonic range tested is larger than the spread in the

Table 10 Parameters for case $\mathrm{T} 1$

\begin{tabular}{lc}
\hline \hline Parameter & Value \\
\hline$M$ & 0.9963 \\
$d_{1-3}$ & $35.3,-18.8,-10.7$ \\
$\omega_{1-3}, \mathrm{rad} / \mathrm{s}$ & $75.1,244.0,474.7$ \\
$L_{1}$ error $\left(C_{l}, C_{d}, C_{m}\right), \%$ & $4.45,9.43,5.25$ \\
\hline \hline
\end{tabular}

subsonic range. For example, when an $M_{\text {step }}$ value of 1.1 is used to calculate the ROM for an $M_{\text {sim }}$ value of 3, the difference between the two is 1.9 Mach number units, which is over twice the entire span of the subsonic Mach range. This would also explain the different trend seen for $M_{\text {sim }}=1.1$. As $M_{\text {step }}$ increases, the $M_{\text {sim }}-M_{\text {step }}$ gap is constantly increasing. For all other cases, as $M_{\text {step }}$ increases, the gap is either decreasing or decreasing at first before increasing. This suggests the expected result that the errors will eventually increase as $M_{\text {step }}$ moves away from $M_{\text {sim }}$.

\section{B. Lift Coefficient Results}

The next series of results shows the lift coefficient results for these test cases. Consider first Fig. 37 , which shows the lift coefficient errors for test cases in which $M_{\text {sim }}=M_{\text {step }}$; it corresponds to Fig. 34, which shows the drag coefficient results over the same cases. The main item to note is that the errors for the lift coefficients are much smaller than those for the drag, with a maximum mean error in the subsonic range of under $3 \%$ and in the supersonic range of under $1.5 \%$. Also, the supersonic errors are smaller than the subsonic errors, continuing the trend seen from the drag coefficient results.

Next, consider Fig. 38, which shows the lift coefficient ROM errors in which the ROMs at each value of $M_{\text {sim }}$ have been calculated using each of the different values of $M_{\text {step }}$. This corresponds to the drag coefficient results shown in Fig. 35. Unlike the drag coefficient results, the lift coefficient errors do show a significant increase over the errors calculated using only $M_{\text {sim }}=M_{\text {step }}$, increasing to maximum mean error of around $7 \%$ for the subsonic cases and $3 \%$ for the supersonic cases. Taking a closer look at Fig. 38a, the errors are largest for the extreme values of $M_{\text {sim }}$ and smallest for those in the middle of the range. This result makes sense when looking at it in terms of the gap between $M_{\text {sim }}$ and $M_{\text {step }}$. As has been shown previously, the ROM errors will eventually increase as $M_{\text {step }}$ continues to move away from $M_{\text {sim }}$; thus, the errors in general will increase with increasing $M_{\text {sim }}-M_{\text {step }}$ gap. The values of $M_{\text {sim }}$ on the edges of the Mach range will have the largest mean values of this $M_{\text {sim }}-M_{\text {step }}$ gap because some of the ROMs will be calculated using an $M_{\text {step }}$ value at or near the other edge of the range. However, values in the middle will have lower $M_{\text {sim }}-M_{\text {step }}$ gap values due to the central location. As a result of this, the $M_{\text {sim }}$ values in the middle of the Mach range in the plot have the lowest errors.

Table 11 Parameter ranges for subsonic/supersonic test cases

\begin{tabular}{lcc}
\hline \hline Parameter & Minimum & Maximum \\
\hline$M$ (subsonic/supersonic) & $0.3 / 1.1$ & $0.9 / 3.0$ \\
$d_{1}$ & -50 & 50 \\
$d_{2}$ & -35 & 35 \\
$d_{3} / \mathrm{rad} / \mathrm{s}$ & -30 & 30 \\
$k$ (subsonic/supersonic) & 63 & 350 \\
\hline \hline
\end{tabular}




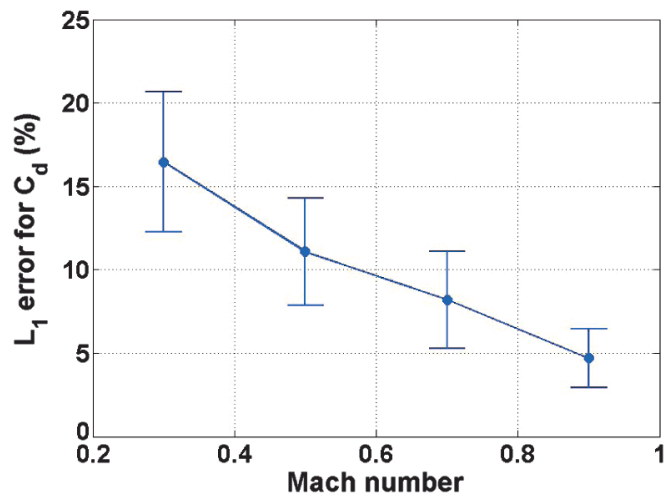

a) Subsonic mach range

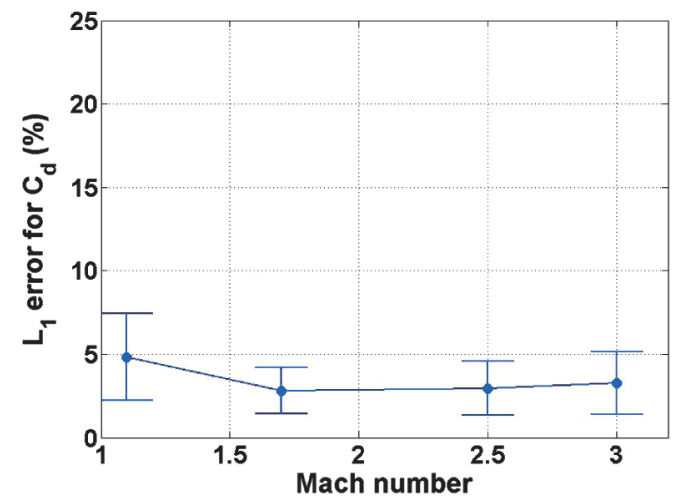

b) Supersonic mach range

Fig. 34 Drag coefficient errors for $M_{\text {sim }}=M_{\text {step }}$.

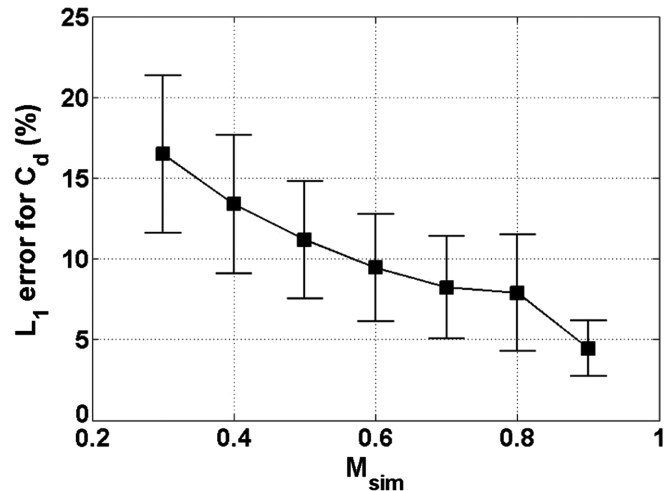

a) Subsonic mach range

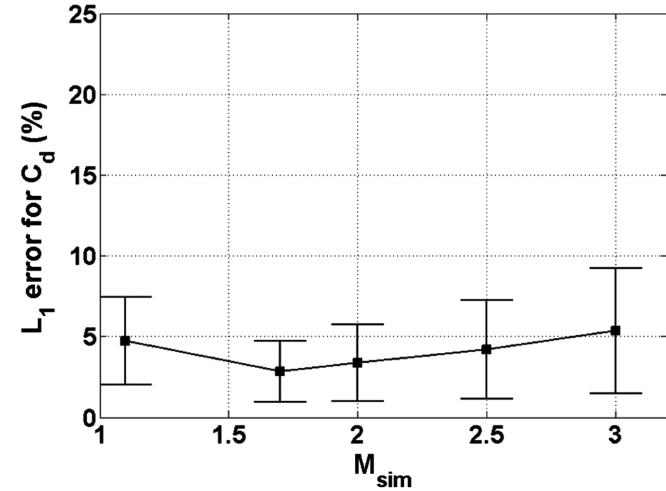

b) Supersonic mach range

Fig. 35 Drag coefficient errors for $M_{\text {sim }}$ using all values of $M_{\text {step }}$.
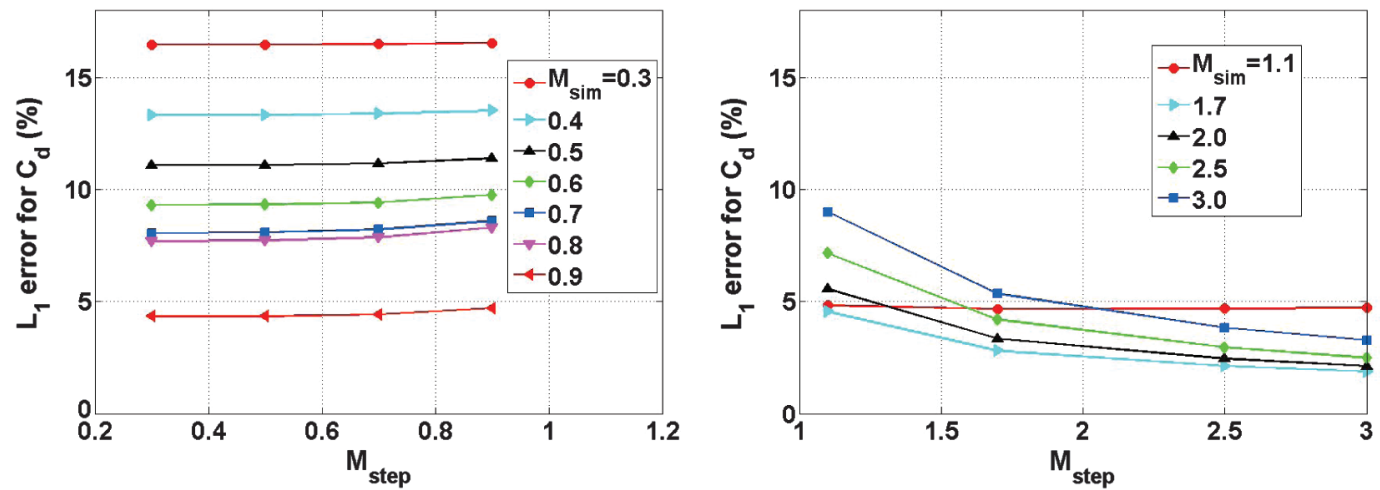

b) Supersonic mach range

Fig. 36 Drag coefficient errors as functions of $M_{\text {step }}$.

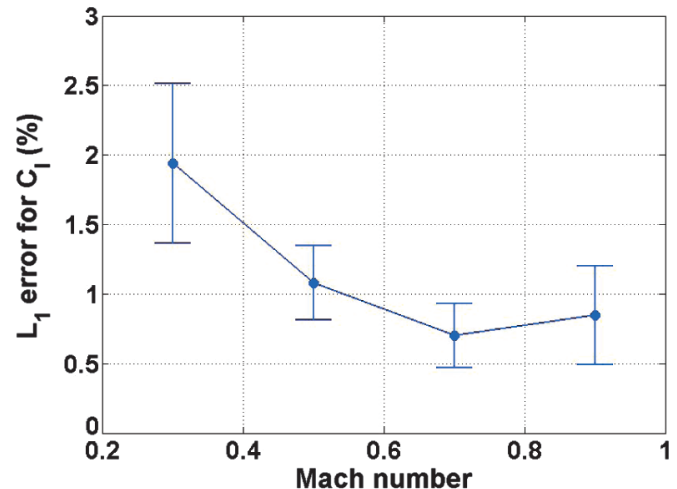

a) Subsonic mach range

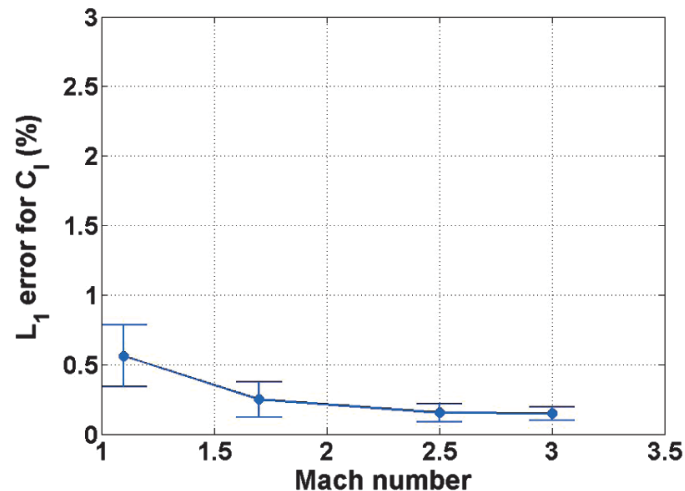

b) Supersonic mach range

Fig. 37 Lift coefficient errors for $M_{\text {sim }}=M_{\text {step }}$. 
This trend of lift coefficient errors being a function of $M_{\text {sim }}-M_{\text {step }}$ gap can be further seen by examining Fig. 39, which is the lift coefficient plot corresponding to the drag results displayed in Fig. 36. As before, each data point corresponds to the mean error of all test cases at a specific value of $M_{\text {sim }}$ with a ROM constructed at a certain value of $M_{\text {step }}$. In Fig. 39a, the data point with the least error for each of the values of $M_{\text {sim }}$ is either found at $M_{\text {step }}=M_{\text {sim }}$ or, for cases in which an exact corresponding value of $M_{\text {step }}$ is not calculated, very close to that value. The trend continues in the supersonic Mach range for all points in Fig. $39 \mathrm{~b}$ as well.

The trends and error values of the moment coefficient reflect those of the lift coefficient for these test cases and thus are not presented here.

\section{Effect of Kriging Surface Sampling Points}

The final item to investigate is how the errors change with the number of sampling points. Consider Fig. 40, which shows the drag coefficient errors for each value of $M_{\text {sim }}$ in both the subsonic (Fig. 40a) and supersonic (Fig. 40b) ranges as functions of the number of sampling points used in correction factor kriging surface construction. For this plot, the ROM-CFD comparisons at each value of $M_{\text {sim }}$ are made for each value of $M_{\text {step }}$. For a point of reference, the rightmost values on the plots, corresponding to over 500 sampling points being used, are the same values as plotted in Fig. 35 .

The data do not show any significant decrease in errors from the fewest to most sampling points for the subsonic range. Slight initial decreases are seen for the values of $M_{\text {sim }} \leq 0.5$ as the number of sampling points increases up to around 300 before leveling off. For the supersonic range, slight error decreases again are seen over the sampling point range. An uptick in the errors is seen at just over 200 sampling points. One possible explanation for this is that the kriging fit for that particular set of parameters caused some type of undulations in the kriging surface that is not seen in reality. However, despite that uptick, the errors for the supersonic range remain smaller than the subsonic range in general.

Next, consider Fig. 41, which shows the lift coefficient results for the same cases as shown in Fig. 40. For the subsonic range, the errors are essentially constant across the sampling point values considered. Slight initial error decreases are seen in the supersonic cases until around 300 sampling points before the errors level off; the general trends seen for the lift coefficient reflect those observed for the drag coefficient for this case in each of the supersonic and subsonic Mach ranges.

\section{Example Case over Entire Mach-Number Range}

It is now important to show how this methodology can be practically applied to a specific example test case spanning a wide range of Mach numbers. This section evaluates the ROM's performance over the entire span of Mach numbers that have been tested for the AGARD 445.6 wing, from Mach 0.3 to Mach 3. In doing so, the first set of results from near Mach 1 has been integrated with these test cases. In practice, one will not have access to an unlimited number of step responses to use for each different value of $M_{\text {sim }}$. Thus, some method must be chosen for how to best deal with values of $M_{\text {sim }}$ that fall somewhere between the different values of $M_{\text {step }}$.

For this section, suppose that the values of $M_{\text {step }}$ found in the first row of Table 12 are the only values for which step responses are available. The goal is to compute ROM results having the least error possible for the $M_{\text {sim }}$ values found in the second row of Table 12. The test cases used here are the same as those used in results presented previously, where, for each value of $M_{\text {sim }}, 25$ separate simulations are conducted. These 25 test cases have the same modal amplitudes and oscillations for each value of $M_{\text {sim }}$ (the parameter space is shown in Table 11), and so the only variation among the tests at different Mach numbers is $M_{\text {sim. }}$. Each data point in the following results represents the mean $L_{1}$ error value over all 25 cases.

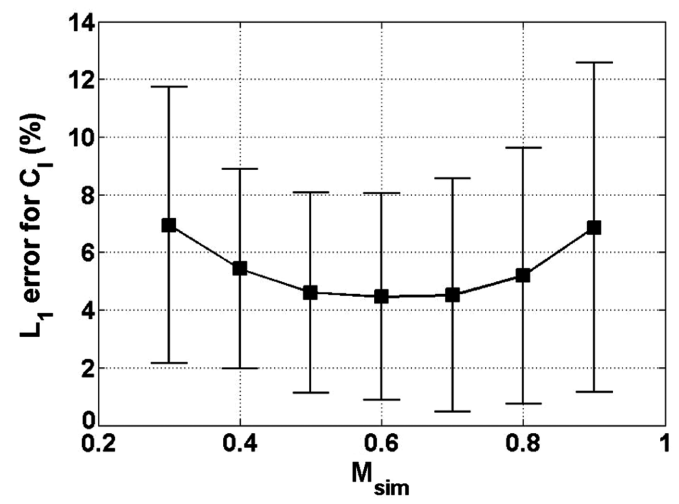

a) Subsonic mach range

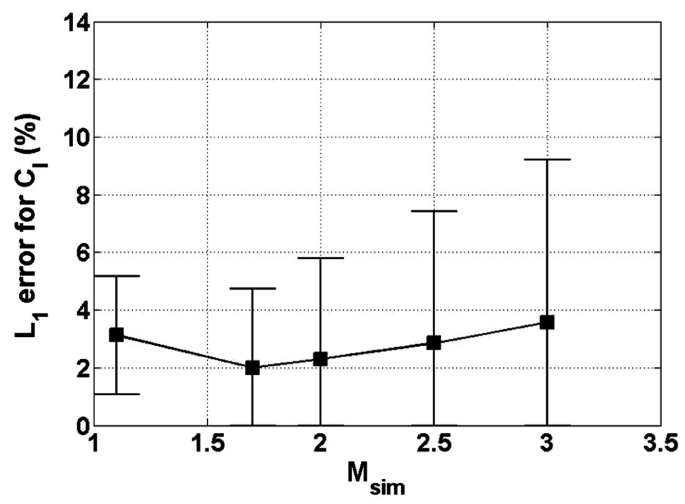

b) Supersonic mach range

Fig. 38 Lift coefficient errors for $M_{\text {sim }}$ using all values of $M_{\text {step }}$.

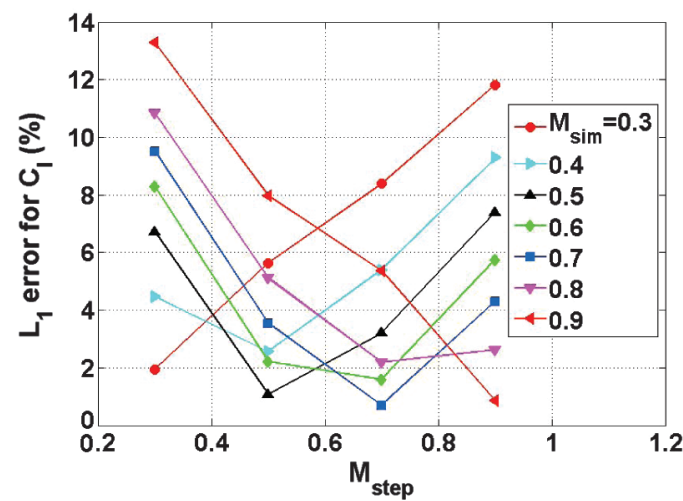

a) Subsonic mach range

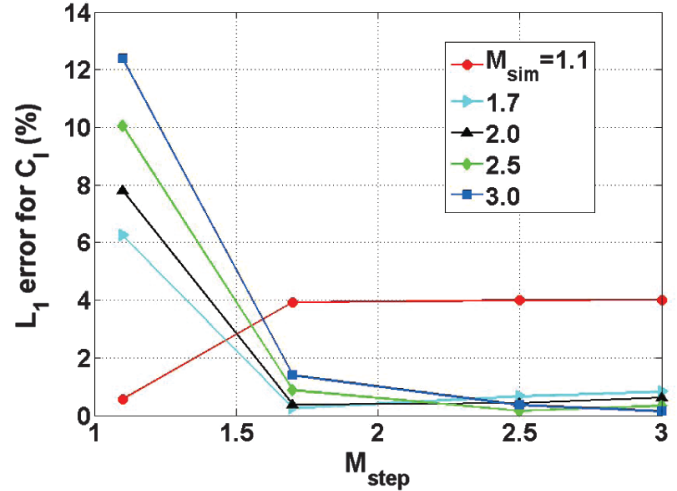

b) Supersonic mach range

Fig. 39 Lift coefficient errors as functions of $M_{\text {step }}$. 


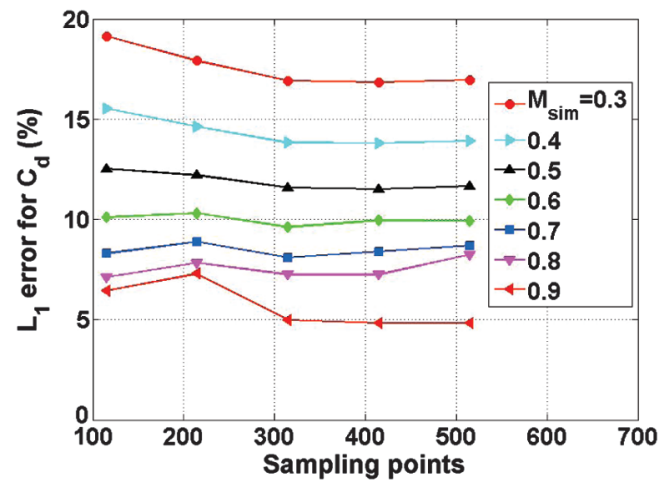

a) Subsonic

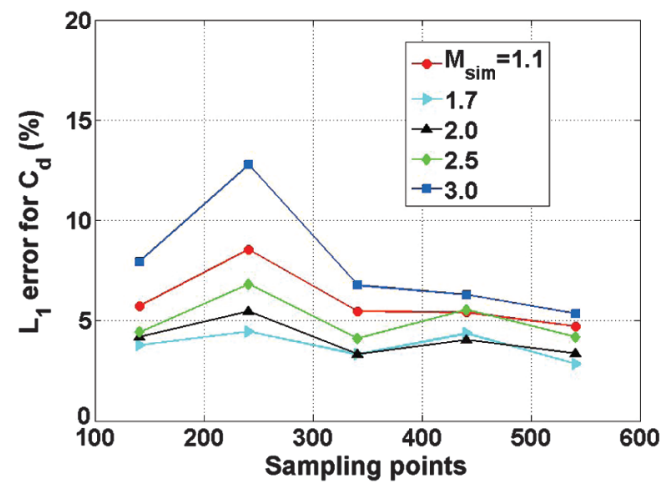

b) Supersonic

Fig. $40 C_{d}$ errors as function of number of sampling points.

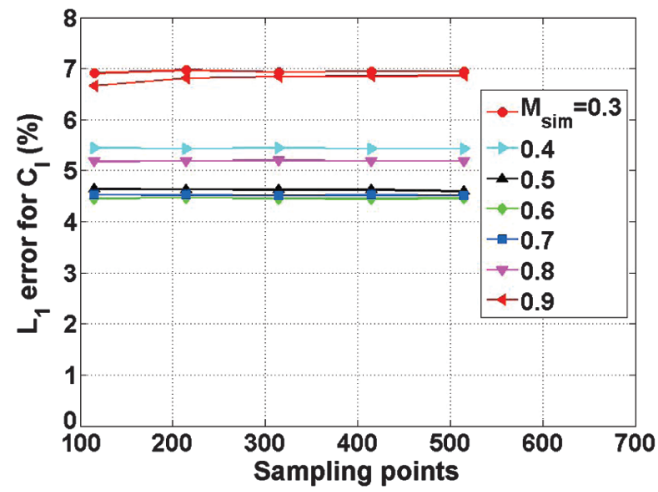

a) Subsonic

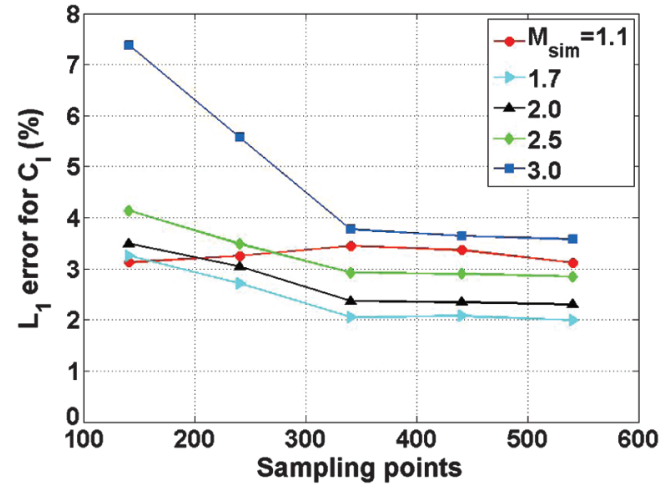

b) Supersonic

Fig. $41 C_{l}$ errors as function of number of sampling points.

For each test case, the ROM is calculated using both methods 1 and 2 , which determine the value(s) of $M_{\text {step }}$ to use when $M_{\text {sim }} \neq M_{\text {step }}$ and were depicted previously in Figs. 9-11. All ROMs in this section use kriging surface sampling points calculated directly from CFD simulations (ROM C). The lift, drag, and moment coefficient results of the tests are shown in Figs. 42-44, respectively, from which several important points can be gathered. First, the lift and moment results show very similar trends in that the errors generally increase as $M_{\text {sim }}$ moves away from $M_{\text {step }}$. The exception to this is the method 1 errors in the subsonic Mach range, which remain relatively steady between $M_{\text {step }}=0.3$ and $M_{\text {step }}=0.9$. To see what happens when another value of $M_{\text {step }}$ is added, consider Fig. $\underline{42 \mathrm{~b}}$, in which $M_{\text {step }}=1.7$ has been added to the list of available step responses (the results in

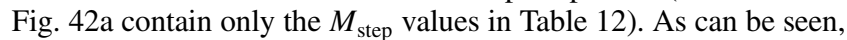
this greatly reduces the error in the supersonic Mach range for each method. In terms of the methods, method 1 (weighted averages) seems to be equal to or better than method 2 in most situations. This is most strikingly seen in the subsonic range around Mach 0.6, where the method 2 error is just above $8 \%$ for the lift coefficient, whereas the method 1 error is down to just over $2 \%$; the corresponding moment coefficient errors at the same location for each of the methods are around $9 \%$ and $3 \%$, respectively. Next, an error spike can be seen for both the lift and moment coefficients around Mach 1, in the transonic regime. Even though the errors do spike, they are still relatively small at just over 5\%. Also, given the nonlinearities present within the transonic regime, a good recommendation would be to have as many values of $M_{\text {step }}$ as feasible within this region.

The drag coefficient results differ in trends from the other two coefficients. Each of the two methods performs similarly throughout the Mach range, and less sensitivity is seen to $M_{\text {step }}-M_{\text {sim }}$ difference. The addition of another $M_{\text {step }}$ value of 1.7 (Fig. $43 \mathrm{~b}$ ) does slightly improve the errors in the supersonic Mach range, though errors were already low to begin with $(\sim 5 \%)$. The results also show that the ROM generally improves in accuracy with Mach number because the Mach 0.3 results show the highest errors. Finally, as with the lift and moment coefficient results, an error spike is seen around Mach 1 in the transonic region, though errors here remain well below the errors at the low end of the Mach-number parameter space around $M=0.3$.

Overall, both methods performed reasonably well, though method 1 is shown to have generally smaller errors for the lift and moment coefficients. For the drag, both methods perform very similarly. Thus, the recommendation for the method of constructing ROMs for situations where $M_{\text {sim }} \neq M_{\text {step }}$ is to use method 1. However, one potential advantage of method 2 over method 1 is computational expense. As mentioned previously, whereas method 2 only requires the computation of one ROM response, method 1 requires the computation of two responses to find the weighted average. In general, the ROM is computationally cheap to compute, and thus this may not be a significant issue. However, if a situation arises in which a very large number of ROM responses will need to be computed, the increased efficiency of method 2 may need to be considered. Finally, a brief word must be given as to how these methods would extend to higher-dimension flight condition parameter spaces. For example, what happens if altitude is considered as well? In this case, method 1 could extend to be the result of a weighted function of the responses from the nearest predetermined number of step response parameter values within the multidimensional parameter space; further work would be required to identify the optimal method for doing so. For method 2, one could still find the nearest set of step response parameter values by calculating the Euclidean distances from the simulation parameters to the various sets of nearby step response parameters.

Table 12 Mach values for example case

\begin{tabular}{cc}
\hline \hline Parameter & Values \\
\hline$M_{\text {step }}$ & $0.3,0.9,1.05,1.1,3.0$ \\
$M_{\text {sim }}$ & $0.3,0.4,0.5,0.6,0.7,0.8,0.9,1.0,1.1,1.7,2.0,2.5,3.0$ \\
\hline \hline
\end{tabular}




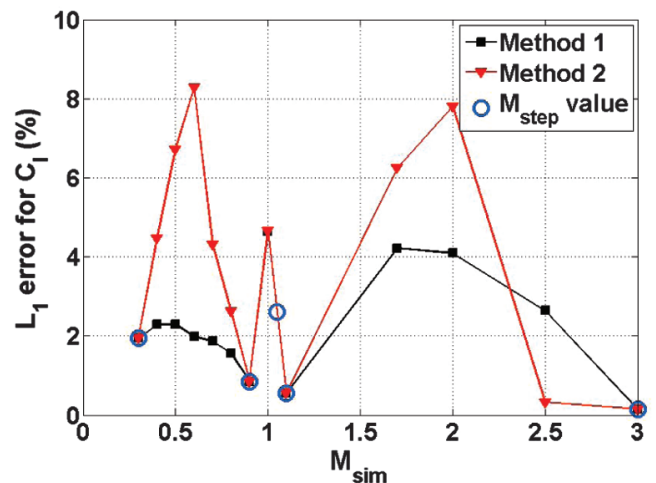

a) $M_{\text {step }}$ values from table 12

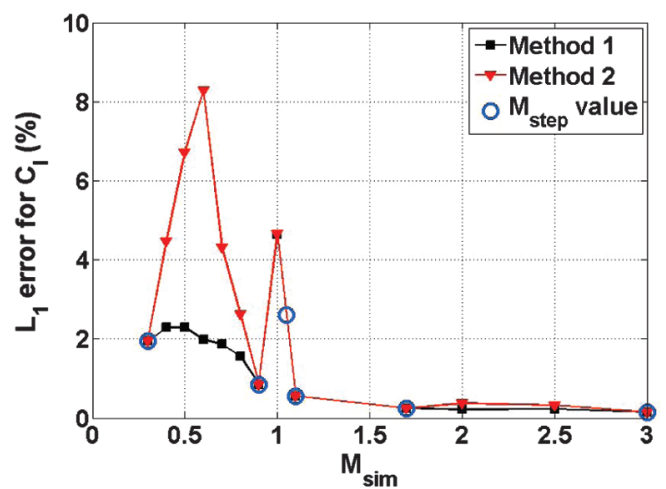

b) Addition of $M_{\text {step }}=1.7$

Fig. 42 Lift coefficient results over entire Mach range.

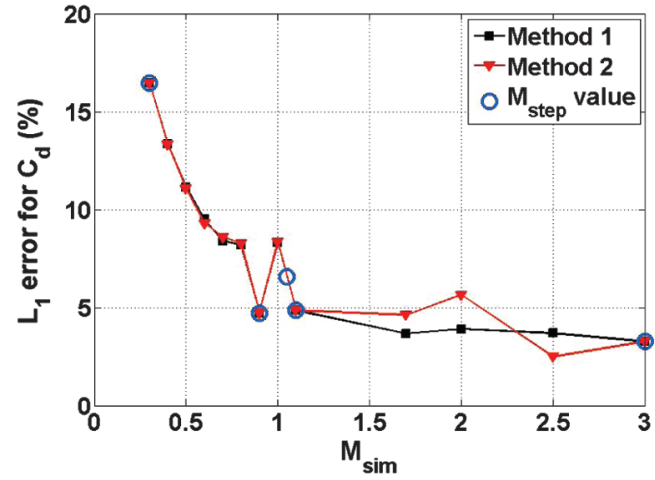

a) $M_{\text {step }}$ values from table 12

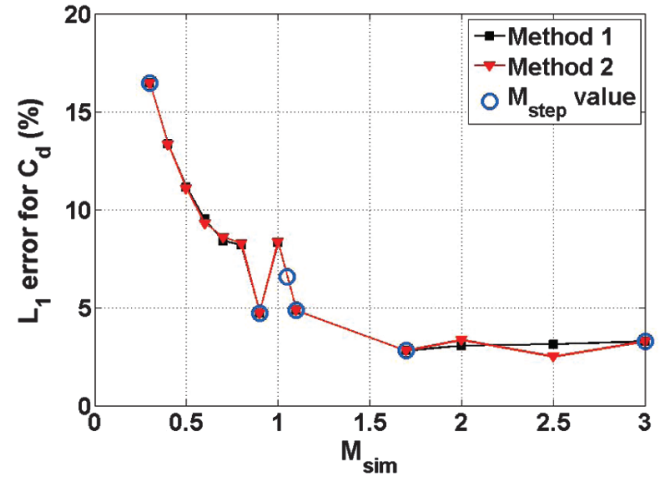

b) Addition of $M_{\text {step }}=\mathbf{1 . 7}$

Fig. 43 Drag coefficient results over entire Mach range.

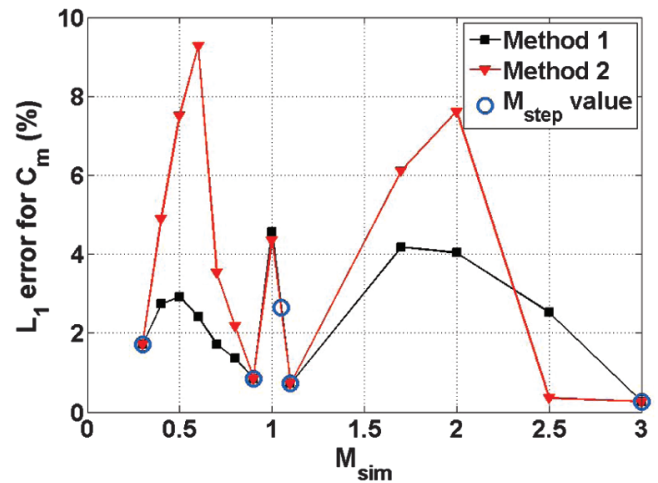

a) $M_{\text {step }}$ values from table 12

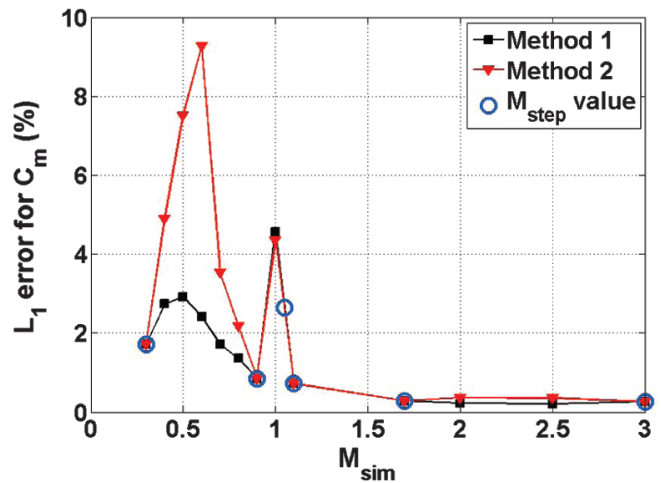

b) Addition of $M_{\text {step }}=1.7$

Fig. 44 Moment coefficient results over entire Mach range.

A mention needs to be made about the potential applicability of the ROM to the extreme low end of the subsonic regime, below Mach 0.3. One factor that would determine how low the ROM should go would be the takeoff speed of the vehicle. While on the ground, vibrations from the wheels, ground effects, special takeoff configurations, and other factors would all influence the dynamics and aerodynamics of the vehicle, thus complicating the analysis. For a point of reference, the takeoff speed of the SR-71 Blackbird is right around $200 \mathrm{kt}$ [33], corresponding to a Mach number of 0.3 assuming $70^{\circ} \mathrm{F}$ ambient temperature. If the ROM is desired to be applied for these low Mach numbers, the ROM errors would be expected to increase with decreasing Mach number.

\section{Computational Savings}

Finally, a quantification of the computational time savings needs to be given between the ROM and full-order CFD simulations. The unsteady sinusoidal CFD simulations were computed using 16 total processors on the NASA Pleiades Supercomputer consisting of Intel Xeon E5-2670, X5670, and X5675 processors with at least $2.6 \mathrm{GHz}$ processor speed. Conversely, the ROM simulations for the same cases were conducted on a desktop computer with a $2.66 \mathrm{GHz}$ Intel Core $2 \mathrm{CPU}$ with $3 \mathrm{~GB}$ of RAM. Figure 45 a shows a logarithmic plot of the comparative times between the ROM and CFD runs over a range of Mach numbers; note that the ROM simulation time is per coefficient, and the CFD CPU time is the total time of all processors. Each data point represents the mean value over the same 25 test cases conducted in the previous section at that particular Mach number. Figure $45 \mathrm{~b}$ shows the ratio between the CFD time and the times for each of the ROMs to quantify the improvements. The results show a

Data available online at http://www.nas.nasa.gov/hecc/resources/pleiades .html [retrieved 15 August 2014]. 


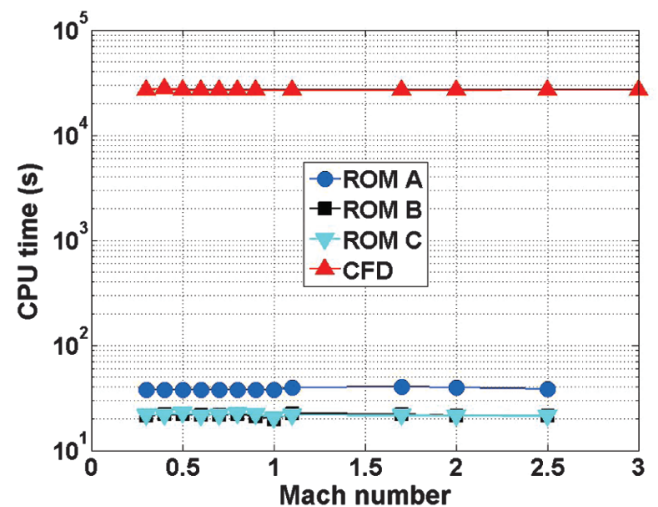

a) CPU times

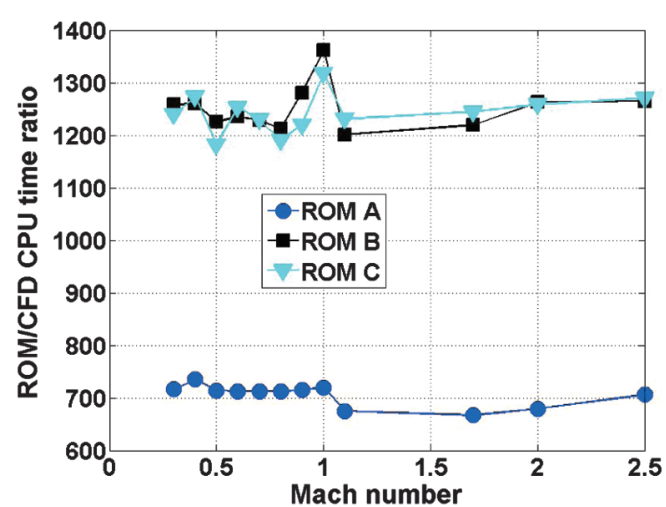

b) CFD/ROM CPU time ratio

Fig. 45 ROM-CFD CPU time comparisons.

roughly three order-of-magnitude decrease in computational cost for the ROMs over the full CFD simulations. Note that the ROM values are the time it takes to run after all upfront computations have been completed. For ROM C, these upfront costs consist of individual CFD runs for each correction factor kriging surface sampling point, whereas for ROMs A and B, these upfront costs only included the steady CFD runs for MoS calculations. Also, between the two MoS ROMs, ROM A has a higher computational cost than ROM B due to the fact that direct calculation of the correction factor takes place at each time step rather than just picking the value off of a kriging surface. Last, note that multiple ROM computations will be necessary to calculate more than one coefficient, and so the computational reduction will be slightly decreased if additional coefficients are calculated. However, for more complex geometries, the CPU time for the full CFD simulations will inevitably increase with the number of grid points, whereas this will have no effect on the ROM solutions. Additionally, it could be possible to find more efficient convolution algorithms, which would improve the computational time of the ROM, making the computational savings even greater.

The preceding discussion compares the ROM and CFD computational time results assuming that the ROM has already been constructed. However, in deciding whether or not to use the ROM methodology (versus the full-order CFD solutions themselves) for a particular problem, the upfront computational cost of the required CFD solutions for ROM construction must be taken into consideration. For example, for the AGARD 445.6 geometry, if only a limited number of full-order simulations would need to be conducted to analyze a certain problem, then it would be much more efficient to simply conduct the full-order CFD simulations rather than go through the ROM construction process because the full-order solutions themselves are not overly computationally expensive in a relative sense for this geometry. However, as the number of simulations to be conducted increases, as in an aeroelastic simulation framework, then the benefits of using the ROM begin to overshadow the brute-force method of direct computation of the full-order solutions. An additional item to take into consideration is the coupling with other codes and analyses. For example, the structural analysis may be performed using a finite-element type of code. Information will need to be passed to the structural analysis from the aerodynamic analysis and vice versa. If the CFD code is incompatible with the other components of the aeroelastic analysis, then the fullorder solutions will not be able to be used for the overall simulation. For each individual problem, given the CPU time and component

Table 13 Relative CPU time for CFD simulations used for ROM construction

\begin{tabular}{lc}
\hline \hline CFD run type & CPU time (\% of total) \\
\hline Steady & 0.3 \\
Steps & 0.9 \\
MoS & 3.6 \\
Correction factor & 95.2 \\
\hline \hline
\end{tabular}

compatibility requirements, the number of simulations that will need to be conducted, and other factors, the cost/benefit analysis of using the ROM versus full-order solutions will be different.

As an example of the ROM construction computational cost, consider the ROM constructed for the subsonic Mach regime from $M=0.3$ to 0.9 . For this ROM, a total of 515 CFD correction factor kriging surface sampling points were computed, along with 77 steady-state runs for MoS calculations and 12 different individual modal step input runs for convolution. The total CPU time for these runs is on the order of $10^{7} \mathrm{~s}$. However, due to parallelization of the CFL3D computations, the actual run time is much less because each simulation is parallelized into 15 separate processors. Table 13 summarizes the relative time requirements, as percentage of total ROM construction CPU time, for each of the necessary types of CFD simulations as applied to the subsonic ROM. As seen in the table, the CFD correction factor sampling point runs account for just over $95 \%$ of this total computational time. However, the MoS CFD computations accounted for only around $3.6 \%$ of the total CPU time, and so using these runs to limit the number of correction factor sampling points can play a significant role in reducing ROM construction expense. For this Mach regime, rather than using a quantifiable errorbased stopping criterion for sampling point determination, separate ROMs were constructed using anywhere from 100 to 500 sampling points, as described previously. The tests showed that, for the most part, using only around 100 sampling points would have been sufficient for this ROM, and doing so would have eliminated the need for around $80 \%$ of the correction factor CFD runs, significantly reducing the time needed for ROM construction. A quantifiable stopping criterion which captures the sufficiency of the $\sim 100$ sampling points using the computationally inexpensive MoS thus is extremely useful to have.

\section{Conclusions}

A reduced-order modeling methodology for use in a hypersonic vehicle controls simulation framework has been applied to the sub-, trans-, and supersonic Mach regimes. This is necessary due to the fact that hypersonic vehicles will accelerate through these Mach regimes on the way up to hypersonic flight. In a controls framework, it is important to be able to use a single aerodynamic model for all calculations regardless of the specific flight conditions because this increases the efficiency of the simulation by avoiding the added computational expense of switching one model out for another at various points throughout the simulation. Separate investigations have been conducted comparing ROM results with full-order CFD simulations in the sub-, trans-, and supersonic Mach regimes. Also, the method-of-segments simplified model has been developed to assist in ROM construction. Finally, a single test case has been followed from low subsonic up to supersonic flight to give a more practical picture of how the ROM can be used in practice. The major conclusions are as follows.

1) For tests with a single mode of oscillation, the ROM methodology in general works well. The ROM errors remain small as 
oscillation amplitude increases with other variables being held constant. However, the drag coefficient errors do increase with oscillation frequency at a larger rate than in the hypersonic regime, an effect that is an artifact of a phase shift between the ROM and CFD results that develops at higher oscillation frequencies.

2) The ROM methodology works well over the range of multimodal oscillation test cases near Mach 1 considered here, having a mean drag coefficient $L_{1}$ error of just over $7 \%$. The other coefficients show generally less errors than the drag.

3) Errors generally decrease with increasing Mach number in both the subsonic and supersonic regimes for the drag coefficient results. The highest errors are generally seen at Mach 0.3. Because of these increasing errors, the reduced frequencies/Mach numbers at this end of the parameter space form a general boundary of where the ROM can be expected to be applicable.

4) MoS generally performs better in the supersonic regime than the subsonic regime due to the smaller reduced frequency values here.

5) Increasing the number of kriging surface sampling points in most cases does not significantly affect the accuracy of the results, showing that a sufficient number of points has been reached.

6) For cases in which $M_{\text {sim }} \neq M_{\text {step }}$, using a method of weighted averages of ROMs computed from neighboring values of $M_{\text {step }}$ successfully reduces the ROM errors, though the method of using the nearest $M_{\text {step }}$ value also works well in certain situations.

7) For the AGARD 445.6 wing, computational savings of well over two orders of magnitude are achieved by using the ROM over full-order CFD solutions.

\section{Acknowledgments}

Funds for the University of Michigan-U.S. Air Force Research Laboratory Collaborative Center in Control Science were made available from the U.S. Air Force Research Laboratory Air Vehicles Directorate grant FA 8650-07-2-3744 (program manager Michael Bolender). Funds were also made available through NASA award NNX08AB32A (program managers Don Soloway and Jorge Bardina) along with computer resources from the NASA Advanced Supercomputing Division. The authors would like to thank Walt Silva from NASA Langley Research Center for providing the computational grid as well as his assistance in the computational studies.

\section{References}

[1] Skujins, T., and Cesnik, C. E. S., "Reduced-Order Modeling of Hypersonic Vehicle Unsteady Aerodynamics," AIAA Atmospheric Flight Mechanics Conference, AIAA Paper 2010-8127, Aug. 2010.

[2] Silva, W., "Discrete-Time Linear and Nonlinear Aerodynamic Impulse Responses for Efficient CFD Analyses," Ph.D. Dissertation, College of William and Mary, Williamsburg, VA, Dec. 1997.

[3] Silva, W., "Identification of Nonlinear Aeroelastic Systems Based on the Volterra Theory: Progress and Opportunities," Nonlinear Dynamics, Vol. 39, No. 1, 2005, pp. 25-62. doi:10.1007/s11071-005-1907-z

[4] Volterra, V., and Fantappie, L., Theory of Functionals and of Integral and Integro-Differential Equations, Blackie and Son, London, 1930.

[5] Rugh, W., Nonlinear System Theory: The Volterra/Wiener Approach, Johns Hopkins Univ. Press, Baltimore, MD, 1981.

[6] Baumann, W., Herdman, T., and Stalford, H., "Recent Work Using Volterra Series as a Methodology to Analyze Nonlinear Aircraft Dynamic Properties," Mathematical and Computer Modeling, Vol. 11, No. 1, 1988, pp. 883-888. doi:10.1016/0895-7177(88)90620-6

[7] Silva, W., and Bartels, R., "Development of Reduced-Order Models for Aeroelastic Analysis and Flutter Prediction Using the CFL3Dv6.0 Code," Journal of Fluids and Structures, Vol. 19, No. 6, 2004, pp. 729 745 . doi:10.1016/j.jfluidstructs.2004.03.004

[8] Silva, W., "Simultaneous Excitation of Multiple-Input/Multiple-Output CFD-Based Unsteady Aerodynamic Systems," Journal of Aircraft, Vol. 45, No. 4, 2008, pp. 1267-1274. doi: $10.2514 / 1.34328$

[9] Kim, T., "Efficient Reduced-Order System Identification for Linear Systems with Multiple Inputs," AIAA Journal, Vol. 43, No. 7, July 2005, pp. $1455-1464$.

doi: $10.2514 / 1.11225$

[10] Kim, T., Hong, M., Bhatia, K., and SenGupta, G., "Aeroelastic Model Reduction for Affordable Computational Fluid Dynamics-Based Flutter Analysis," AIAA Journal, Vol. 43, No. 12, Dec. 2005, pp. 2487-2495. doi: $10.2514 / 1.11246$

[11] Gaitonde, A., and Jones, D., "Reduced Order State-Space Models from the Pulse Responses of a Linearized CFD Scheme," International Journal for Numerical Methods in Fluids, Vol. 42, No. 6, 2003, pp. 581606. doi:10.1002/(ISSN)1097-0363

[12] Allen, C., Taylor, N., Fenwick, C., Gaitonde, A., and Jones, D., “A Comparison of Full Non-Linear and Reduced Order Aerodynamic Models in Control Law Design Using a Two-Dimensional Aerofoil Model," International Journal for Numerical Methods in Engineering, Vol. 64, No. 12, 2005, pp. 1628-1648. doi:10.1002/(ISSN)1097-0207

[13] Singh, R., and Baeder, J., "Direct Calculation of Three-Dimensional Indicial Lift Response Using Computational Fluid Dynamics," Journal of Aircraft, Vol. 34, No. 4, 1997, pp. 465-471. doi: $10.2514 / 2.2214$

[14] Lind, R., Prazenica, R., Brenner, M., and Baldelli, D., "Identifying Parameter-Dependent Volterra Kernels to Predict Aeroelastic Instabilities," AIAA Journal, Vol. 43, No. 12, Dec. 2005, pp. 2496-2502. doi:10.2514/1.12042

[15] Prazenica, R., Reisenthel, P., Kurdila, A., and Brenner, M., "Volterra Kernel Extrapolation for Modeling Nonlinear Aeroelastic Systems at Novel Flight Conditions," Journal of Aircraft, Vol. 44, No. 1, 2007, pp. 149-162. doi: $10.2514 / 1.22764$

[16] Omran, A., and Newman, B., "Piecewise Global Volterra Nonlinear Modeling and Characterization for Aircraft Dynamics," Journal of Guidance, Control, and Dynamics, Vol. 32, No. 3, 2009, pp. 749-759. doi: $10.2514 / 1.40655$

[17] Omran, A., and Newman, B., "Full Envelope Nonlinear ParameterVarying Model Approach for Atmospheric Flight Dynamics," Journal of Guidance, Control, and Dynamics, Vol. 35, No. 1, 2012, pp. 270-283. doi: $10.2514 / 1.51577$

[18] Silva, W., "Recent Enhancements to the Development of CFD-Based Aeroelastic Reduced-Order Models," 48th AIAA/ASME/ASCE/AHS/ ASC Structures, Structural Dynamics, and Materials Conference, AIAA Paper 2007-2051, April 2007.

[19] Bisplinghoff, R. L., Ashley, H., and Halfman, R. L., Aeroelasticity, Addison-Wesley, Cambridge, MA, 1955, p. 813.

[20] Raveh, D., "Reduced-Order Models for Nonlinear Unsteady Aerodynamics," AIAA Journal, Vol. 39, No. 8, Aug. 2001, pp. 1417-1429. doi:10.2514/2.1473

[21] Matheron, G., "Principles of Geostatistics," Economic Geology, Vol. 58, No. 8, 1963, pp. 1246-1266. doi:10.2113/gsecongeo.58.8.1246

[22] Lophaven, S., Nielsen, H., and Sondergaard, J., "DACE: A MATLAB Kriging Toolbox, Version 2.0," Informatics and Mathematical Modelling TR-2002-12, Denmark, Aug. 2002.

[23] McKay, M., Beckman, R., and Conover, W., "A Comparison of Three Methods for Selecting Values of Input Variables in the Analysis of Output from a Computer Code," Technometrics, Vol. 21, No. 2, May 1979, pp. 239-245. doi: $10.2307 / 1268522$

[24] Yates, E. C., Jr., "AGARD Standard Aeroelastic Configurations for Dynamic Response. Candidate Configuration 1-Wing 445.6," NASA TM-100492, 1987.

[25] Raveh, D., Levy, Y., and Karpel, M., "Efficient Aeroelastic Analysis Using Computational Unsteady Aerodynamics," Journal of Aircraft, Vol. 38, No. 3, 2001, pp. 547-556. doi: $10.2514 / 2.2795$

[26] Raveh, D., "Identification of Computational-Fluid-Dynamics Based Unsteady Aerodynamic Models for Aeroelastic Analysis," Journal of Aircraft, Vol. 41, No. 3, 2004, pp. 620-632. doi:10.2514/1.3149

[27] Cowan, T. J, Arena, A. S., and Gupta, K., "Accelerating Computational Fluid Dynamics Based Aeroelastic Predictions Using System Identification," Journal of Aircraft, Vol. 38, No. 1, 2001, pp. 81-87. doi: $10.2514 / 2.2737$

[28] Munteanu, S., Rajadas, J., and Chattopadhyay, A., "Nonlinear Aeroelastic Analysis Using a CFD-Based State Space ROM," 47th AIAA/ASME/ASCE/AHS/ASC Structures, Structural Dynamics, and Materials Conference, AIAA Paper 2006-2024, May 2006.

[29] Silva, W., and Raveh, D., "Development of Unsteady Aerodynamic State-Space Models from CFD-Based Pulse Responses," 42nd AIAA/ 
ASME/ASCE/AHS/ASC Structures, Structural Dynamics, and Materials Conference and Exhibit, AIAA Paper 2001-1213, April 2001.

[30] Raveh, D., Levy, Y., and Karpel, M., "Aircraft Aeroelastic Analysis and Design Using CFD-Based Unsteady Loads," 41st AIAA/ASME/ASCE/ AHS/ASC Structures, Structural Dynamics, and Materials Conference and Exhibit, AIAA Paper 2000-1325, April 2000.
[31] Krist, S. L., Biedron, R. T., and Rumsey, C. L., "CFL3D User's Manual (Ver. 5.0)," NASA TM-1998-208444, 1998.

[32] Brigham, E., The Fast Fourier Transform and Its Applications, Prentice-Hall, Englewood Cliffs, NJ, 1988.

[33] Shul, B., Sled Driver: Flying the World's Fastest Jet, 3rd ed., Mach 1, Inc., Chico, CA, 1991, p. 50. 